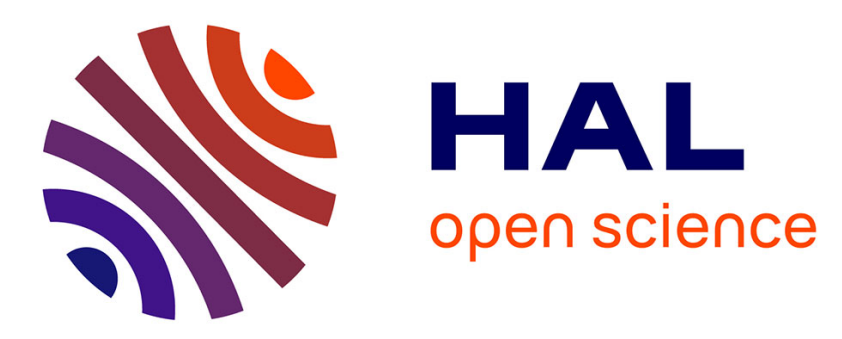

\title{
Eulerian Moment Methods for Automotive Sprays
}

Oguz Emre, Damien Kah, Stéphane Jay, Quang-Huy Tran, Anthony Velghe, Stéphane de Chaisemartin, Frédérique Laurent, Marc Massot

\section{To cite this version:}

Oguz Emre, Damien Kah, Stéphane Jay, Quang-Huy Tran, Anthony Velghe, et al.. Eulerian Moment Methods for Automotive Sprays. Atomization and Sprays, 2015, 25 (3), pp.189-254. 10.1615/AtomizSpr.2015011204 . hal-01097531

\section{HAL Id: hal-01097531 \\ https://hal-ifp.archives-ouvertes.fr/hal-01097531}

Submitted on 19 Dec 2014

HAL is a multi-disciplinary open access archive for the deposit and dissemination of scientific research documents, whether they are published or not. The documents may come from teaching and research institutions in France or abroad, or from public or private research centers.
L'archive ouverte pluridisciplinaire HAL, est destinée au dépôt et à la diffusion de documents scientifiques de niveau recherche, publiés ou non, émanant des établissements d'enseignement et de recherche français ou étrangers, des laboratoires publics ou privés. 


\title{
Eulerian Moment Methods for Automotive Sprays
}

\author{
O. Emre ${ }^{1,2,3}$, D. Kah ${ }^{1,2,3}$, S. Jay ${ }^{* 1}$, Q.-H. Tran ${ }^{1}$, A. Velghe ${ }^{1}$, S. de Chaisemartin ${ }^{1}$, R.O. Fox ${ }^{2,3}$, \\ F. Laurent ${ }^{2,3,4}$, M. Massot ${ }^{2,3,4}$
}
1: IFP Energies nouvelles, 1,4 av. Bois Préau, 92852 Rueil-Malmaison, France ; Institut Carnot IFPEN Transports Energie Stephane.Jay@ifpen.fr

\author{
2: CNRS UPR288, Laboratoire EM2C, 92295 Châtenay-Malabry, France \\ 3: Ecole Centrale Paris, 92295 Châtenay-Malabry, France \\ 4: Fédération mathématique de l'Ecole Centrale Paris - FR CNRS 3487 \\ Marc.Massot@ecp.fr
}

\begin{abstract}
To assist industrial engine design, 3D simulations are increasingly used as they allow evaluation of a wide range of engine configurations and operating conditions and bring a comprehension of the underlying physics complementary to experiments. While the gaseous flow description has reached a certain level of maturity, the multiphase flow description involving the liquid jet fuel injected into the chamber still faces some major challenges. There is a pressing need for a spray model that is time efficient and accurately describes the fuel-particle cloud dynamics downstream of the injector, which is an essential prerequisite for predictive combustion simulations. Due to the highly unsteady nature of the flow following the high-pressure injection process and the complexity of the flow regimes from separated/dense compressible phases to fully developed turbulent spray with evaporating droplets, Eulerian-Eulerian descriptions of two-phase flows are seen as very promising approaches towards realistic and predictive simulations of the mixing process. However they require some effort in terms of physical modeling and numerical analysis related to the more complex mathematical structure of the system of equations and to the unclosed terms appearing in space/time-average equations. Among the various challenges faced, one critical aspect is to capture spray polydispersity in this framework. A review of recent developments that have permitted key advances in the spray modeling community is proposed in this paper. It is divided into four parts. First, an introduction to automotive spray modeling is provided. Then the formalisms for the description of the disperse region of an engine spray are presented with particular emphasis on the pros and cons of classical Lagrangian particle methods versus Eulerian approaches. The third part presents the motivation for and the recent developments of Eulerian high-order moment methods for size polydispersion. Finally, the extension to fully two-way coupled interactions with the gas phase and the implementation of such methods for variable-geometry applications in CFD codes is described in the fourth part. Using realistic direct injection conditions computed with the IFP-C3D solver, the application and efficiency of Eulerian approaches is illustrated.
\end{abstract}

\section{Contents}

1 Introduction $\quad 3$

2 Kinetic spray modeling $\quad \mathbf{7}$

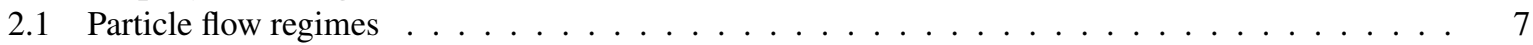

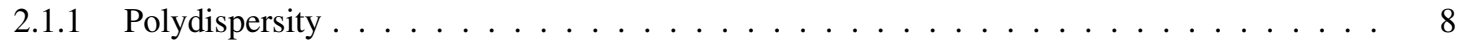

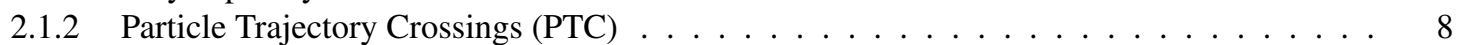

2.2 Williams-Boltzmann kinetic equation: framework and derivation . . . . . . . . . . . 8

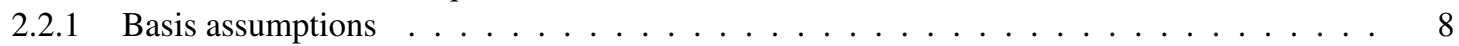

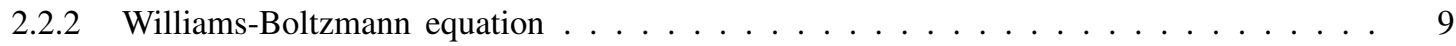

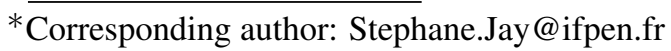




\section{Eulerian moment methods for sprays}

3.1 Derivation strategies in a DNS context : Quadrature vs hydrodynamic velocity equilibrium conditioned in size . . . . . . . . . . . . . . . . . . . . . . . . . 11

3.2 Eulerian polykinetic modeling through kinetic-Based Moment Methods (KBMM) . . . . . . . . . 12

3.3 Kinetic based Eulerian simulation of polydisperse droplets . . . . . . . . . . . . . . . . 13

3.4 Eulerian polydisperse approach with size sampling $\ldots \ldots \ldots \ldots \ldots \ldots \ldots \ldots$

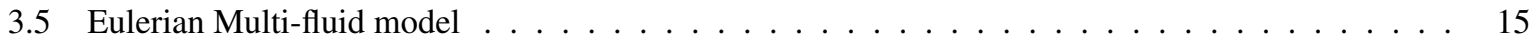

3.5.1 Original MF assumptions and the model derivation . . . . . . . . . . . . . . . 15

3.5.2 MF with two size moments per section . . . . . . . . . . . . . . . . . 16

3.5.3 PTC with MF method . . . . . . . . . . . . . . . . . . . . . . . . . . . . 17

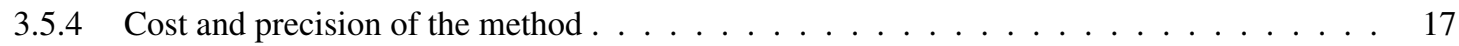

3.5.5 Conclusion on MF method . . . . . . . . . . . . . . . . . . . . . 17

3.6 High order moment methods based on the Maximum entropy NDF reconstruction $\quad \ldots \ldots \ldots$

3.7 Eulerian Multi-Size Moment (EMSM) method . . . . . . . . . . . . . . . . . . . . . . . 18

3.7.1 Derivation of EMSM . . . . . . . . . . . . . . . . . . . . . . . . . . . . . . . . . . 19

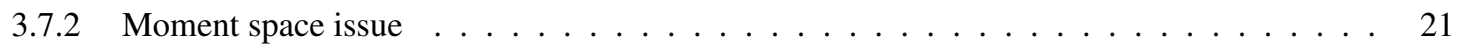

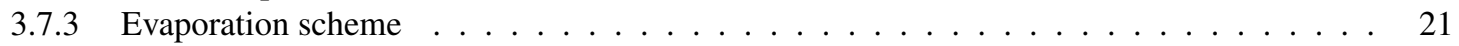

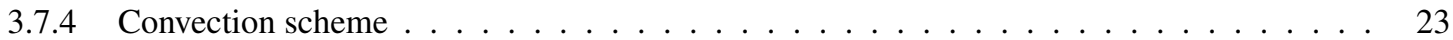

3.7.5 Academic realizations through EMSM method . . . . . . . . . . . . . . . . . 23

3.7.6 Recent extension of EMSM method to capture hetero-PTC: CSVM method . . . . . . . 23

4 Implementation and tests in an engine solver 25

4.1 Adaptation to the Abritrary Lagrangian Eulerian (ALE) formalism . . . . . . . . . . . . . . 25

4.1.1 Overview of peculiarities introduced by the ALE formalism . . . . . . . . . . 25

4.1 .2 Elementary tests . . . . . . . . . . . . . . . . . . . . 25

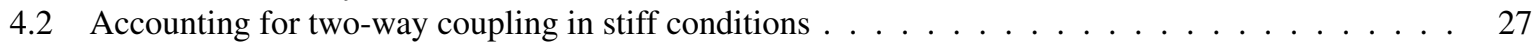

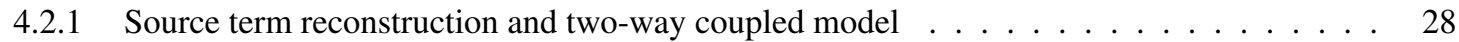

4.2.2 Description of the proposed strategy . . . . . . . . . . . . . . . . . . . . . . . . 29

4.2.3 Evaluation strategy of the method . . . . . . . . . . . . . . . . . . . . . . . . 29

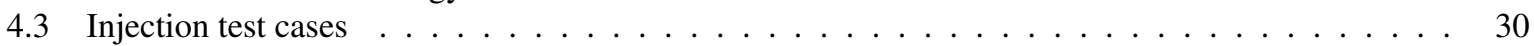

4.3.1 Eulerian spray initialization $\ldots \ldots \ldots \ldots \ldots \ldots \ldots \ldots \ldots \ldots \ldots$

4.3.2 Comparisons between Eulerian and Lagrangian simulations _ . . . . . . . . . . . . 31

4.3.3 High pressure injection conditions . . . . . . . . . . . . . . . . . . . 35

4.4 Concluding remarks on injection simulations $\ldots \ldots \ldots \ldots \ldots \ldots \ldots \ldots$

5 Conclusions and future directions 39

$\begin{array}{ll}\text { References } & 40\end{array}$ 


\section{Introduction}

The transportation sector is a key element for economic development for every country in the world. Fossil fuels (gasoline, diesel, natural gas, ...) are the main source of energy for road transportation, through their use in internal combustion (IC) engines, and will certainly prevail in the near future. In this context, car and engine manufacturers have to face various constraints and expectations, in relation with the engine efficiency and emissions [52] and which are sometimes contradictory:

- Need to reduce significantly green house effect gases, among them $\mathrm{CO}_{2}$. Road transportation is responsible for one third of emissions originating from human activities on earth.

- Reduction of fossil reserves inducing a necessary diversification of fuel origins for internal combustion engines (biofuels, synthesis fuels) and research for alternate solutions (partial or full electrification).

- More stringent regulations on emissions, such as particles, NOx, unburnt hydrocarbons and a growing number of regulated pollutants in the near future.

- Need to increase competitiveness in a challenging worldwide industrial sector.

To meet social demands for more efficient transport that is less polluting and compatible with sustainable development, the automotive industry has to propose new technological solutions. One of these solutions is to introduce hybrid or even pure electrical power units. As compared to classical engines, the efficiency of electrical or hybrid engines is better for urban mobility purposes, however, it shrinks drastically for long distance travel. In this context, it appears that the development of high efficiency thermal engine technologies could represent an important contribution to economically viable and environmentally friendly road transportation. From this point of view, direct injection technologies for both spark and compression ignition engines offer potential benefits in terms of specific power, combustion efficiency and control flexibility compared to more classical injection technologies. The evolution of regulations on pollutant emissions requires much more effort to understand and model physical processes associated with these technologies and their applications.

The increasing complexity of engine systems requires more and more advanced design tools including numerical simulation. Reduction of design delays imposes the use of numerical tools and a reduced number of costly prototypes. Present developments are related to 3D modeling of two-phase flows for which one application is direct fuel injection. The role of modeling is to enable a description of the physical mechanisms in view of understanding and analysis. On the one hand, it gives access to information that is not directly attainable through experiments. On the other hand, the question of validity of the approaches is the central preoccupation with which one has to cope. Figure 1 illustrates schematically (with a few examples of technical solutions related to IC engines) how accounting for engine sprays is at the heart of present issues (pollutant reduction, efficiency improvement, fuel diversification) and which motivated the model developments presented in the following sections.

The presence of a spray in the combustion chamber implies a number of challenges for numerical simulations resulting from the complex liquid phase topology (primary and secondary atomization),coupled physical processes (liquid/gas mass, momentum and energy exchanges) and large temporal and spatial scale variations. This issue is transversal to several injection applications (not only direct injection engines) and a few illustrations of experimental results for practical situations are given in Figure 2. Although the characteristic dimensions and time scales are very different between all of these devices, one can notice some similarities; in particular, on the nature of the interface between the light and the dense fields. One can clearly measure the interest for an Eulerian formulation capable of coherently describing the two-phase flows in such situations.

Modeling approaches have recently benefited from the increase of computational power, permitting to carry out much more detailed simulations of these intricate phenomena. Before entering into the details of the models, it is useful to situate their development in the global context of two-phase flow modeling and in particular that of Eulerian approaches. First, models for disperse two-phase flows are numerous and have been the subject of fundamental and applied studies starting with Lagrangian methods [73]. These approaches are still widely used in industry as they can be easily implemented, have high numerical precision and can cover a range of applications that is sufficiently large. The first type of Lagrangian method is the Discrete Particle Simulation (DPS) which consists of tracking each individual physical droplet in the spray [101, 94, 130]. The effect of particle volume occupation on the surrounding fluid flow is not resolved and the fluid particle global mass, momentum and energy interactions are modeled under the form of a point particle assumption. On the other hand, the Lagrangian Stochastic Parcel (SP) approach has been developed in [93, 31] to supply DPS in configurations where the high number of physical droplets prevents to use one numerical particle for each droplet, given the high computational 


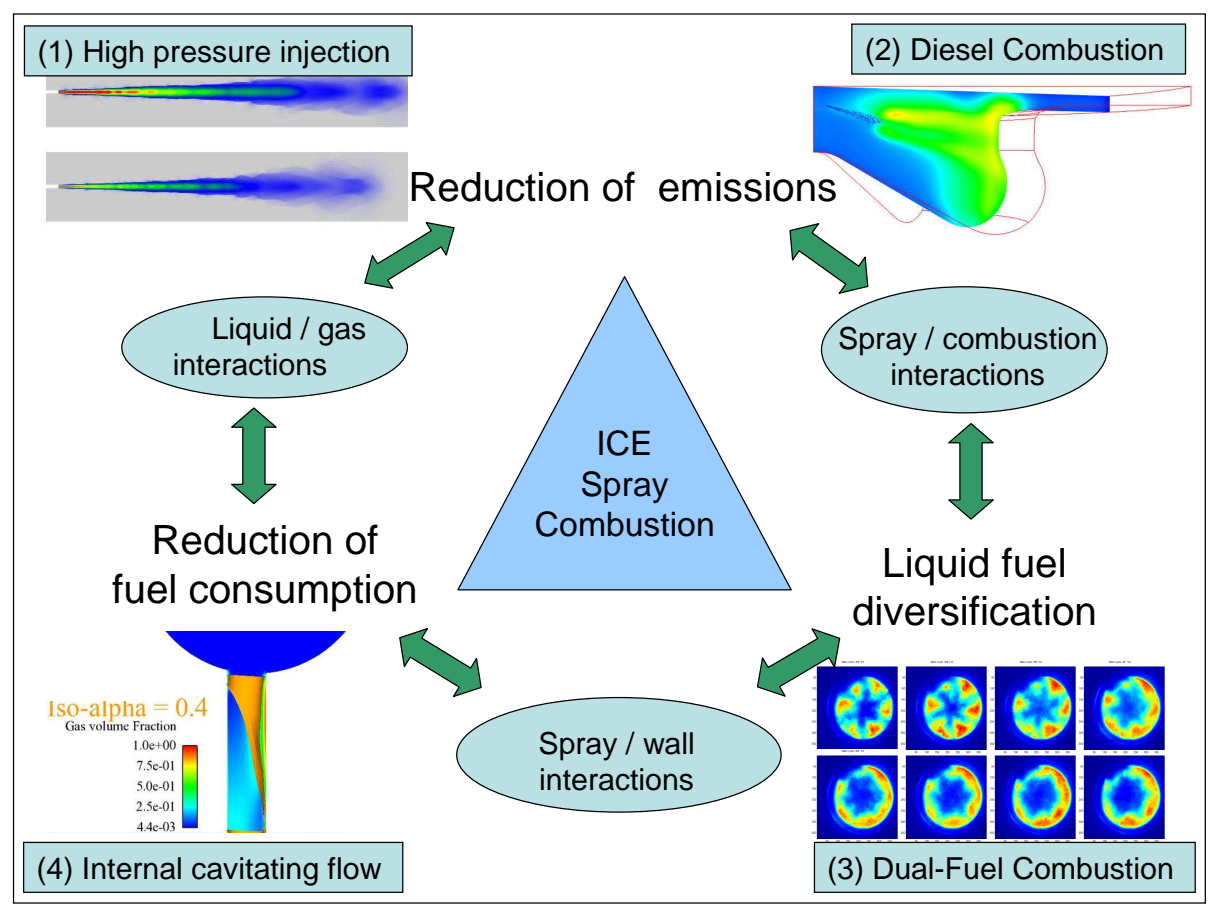

Figure 1. Illustration of the importance of spray formation understanding and modeling for future automotive engine developments in view of reaching an environmentally friendly and economically viable road transportation. Two-phase flows are in the heart of internal combustion engines predictive simulations (internal injector flow, spray/gas turbulent interactions, spray/combustion interactions, spray/wall interactions) as illustrated here. Examples are given for (1) two-way coupled full Eulerian high pressure injection simulations [117], (2) Diesel engine combustion simulation and analysis [77], (3) Dual-Fuel combustion regimes investigations [6, 5] and (4) injector internal cavitating flow simulations [48].

cost associated. In SP, each numerical particle, also called parcel, represents several physical droplets. While DPS and SP methods represent the single realization of a particles flow, the least Lagrangian method called Direct Simulation Monte Carlo (DSMC) provides an other modeling option, describing a statistical representation of the particles flow through ensemble averaging of multiple realizations [109, 110, 8]. In fact, DSMC can be considered as a resolution of William Boltzmann equation based on the kinetic spray modeling [123, 124]. DSMC requires many numerical particles to represent each individual physical droplet, leading higher refinement level than in DPS. On the contrary, the SP method aims at coarsening the DPS description, thus is used to compute industrial configurations. SP is implemented for instance in the Kiva II code [2] and in IFP-C3D code [9]. Despite their frequent use for industrial applications, Lagrangian methods suffer from a certain number of intrinsic limitations. First, they raise the question of the coupling with the Eulerian description of the gas phase. This question is still open since it involves two ways of description that are fundamentally different even if some recent contributions have pave the way to at least obtaining grid convergence of solutions [13]. Moreover, in the framework of domain decomposition for parallel computations it is needed to use complex and costly dynamic partitioning methods, to ensure a good load balancing between different parallel processes. Let us recall also that some advances have been obtained in this field [46]. Finally, in order to describe a full spray injection in a consistent manner, Lagrangian methods, when they are used, have to be coupled with an Eulerian model for separate-phase flows [21]. More recently, Eulerian approaches have undergone rapid development, mainly due to the increase of massively parallel computing. There is a natural interest to use Eulerian descriptions of two-phase flow in the case of separated phases for which Lagrangian methods are not conceptually designed. This kind of approach has been widely developed in the last ten years.

There is a number of models belonging to the class of Eulerian approaches in two-phase flow fluid mechanics. The necessity to understand well the differences, the hypothesis and application conditions is one of the prelim- 

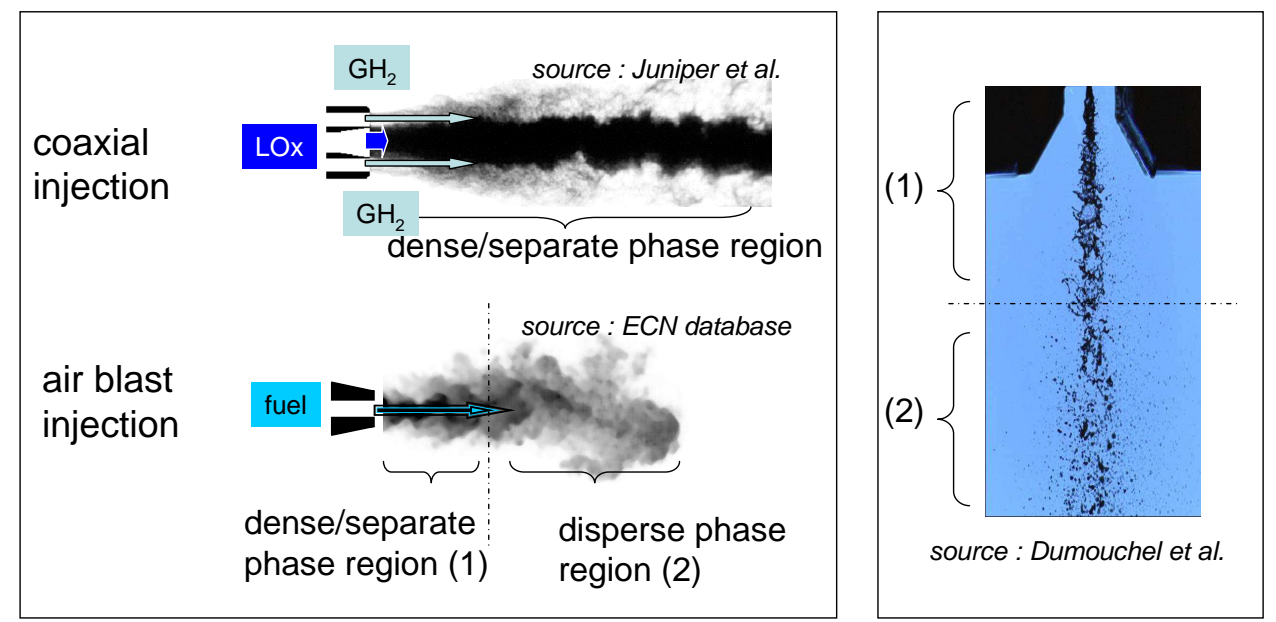

Figure 2. Visualization of the injection region in various practical situations. Left (top): instantaneous ombroscopy visualisation of dense/light oxygen mixture for coaxial injection between gaseous hydrogen $\left(\mathrm{GH}_{2}\right)$ and liquid oxygen (LOx) in transcritical conditions - $P=70$ bar (Juniper et al. [56]). Left (bottom): instantaneous ombroscopy visualisation of mixture fraction in a Diesel jet (Engine Combustion Network data base, Sandia [95]). Right: instantaneous ombroscopy visualisation of injection in an experimental research device for jet atomization in low pressure conditions (Dumouchel et al., [32]).

inary requirements to evaluate the relevance of the new developments described hereinafter. A proposition for a classification of separated and disperse two-phase flows can be found in the PhD thesis of Damien Kah [57]. Some new features of these computational methods are described in the work of Le Chenadec [19, 18]. One can mention approaches where a direct resolution of the interface is envisioned, namely the front tracking [115] and front capturing $[50,51,87]$ approaches. Nevertheless, from a practical point of view, a full direct resolution is still not achievable to predict the overall behavior of a liquid phase from the onset of injection to the fully developed spray formation and evaporation under real engines operating conditions. The use of reduced order models, giving access to

- a precise and stable resolution of the mixture formation statistics at every point of an engine, and

- a coherent description of the full two-phase flow from the internal/close injector region, including all thermodynamically relevant conditions and compressibility effects encountered in practical applications,

is thus a necessity in view of the development of predictive simulation tools.

Phase-average models based on averaged field equations are of particular interest to compute two-phase flows in complex configurations. Fundamental work presenting the derivation of equations associated with this kind of approach was initially proposed in the context of thermohydraulic two-phase flow [54] and the mathematical basis at the beginning of the eighties [29]. Once the field equations are established, closure relations are then necessary for exchange terms between phases. The classification proposed in [57] distinguishes between the following models, the associated hypothesis and the necessary conditions on the mathematical structure and thermodynamic consistency: 
- Mixture models

- Two-fluid models without interface (compressible and incompressible)

- Two-fluid models with interface (compressible and incompressible)

One challenge for this kind of development is to ensure the stability and thermodynamic consistency of the final system of equations. To deal with the physics of compressible two-phase flow that are encountered in engines, there is a number of recent works related to the questions around the Baer \& Nunziato approach [3] and numerical approaches to solve this system $[104,88]$ and more recently $[44,43,67]$. This approach offers a complete description of separated flows phenomena.

The so-called seven equation approach with Baer \& Nunziato closures has been more particularly detailed and is used in the IFP-C3D code [9] to deal with dense flows ([114],[117],[74]). A certain number of modeling difficulties arise and have to be overcome to make this kind of approach operational:

- First, the question of the transition between flow regimes is crucial. Some authors have proposed theoretical developments [71],[90] around the notion of interfacial area based on original ideas in combustion [75],[12] to tackle this issue. A modeled transport equation for interfacial area is used to represent the mixing processes from the internal injector region down to the fully developed spray region [116, 55, 22, 69]. Using these notions, efforts have been made towards the simulation of the entire injection process with Eulerian-Lagrangian coupling (like in the Eulerian LAgrangian Spray Atomization solver [116, 22, 69]) or Eulerian-Eulerian simulations using the two-fluid Baer \& Nunziato approach [3] with two surface density transport equations [74]. These aspects, related to flow regime transitions, will not be considered in the present paper as they constitute the subject of ongoing developments at the present time and, in particular, further improvements are being considered to make this approach fully operational for engine combustion calculations.

- Second, clouds of droplets formed by high pressure injection used in automotive engines are, by nature, polydisperse and one has to introduce a model for the polydispersion of the fully developed spray region, which is not straightforward for standard Eulerian approaches and is critical for the prediction of the vapor phase distribution and for combustion.

This last point is the central point of this paper as it was the thread of several recent works that have addressed some major difficulties to make these approaches able to handle polydispersity $[82,83,60]$ of evaporating sprays for IC engines conditions in a robust and accurate way [35, 58], and which cover:

- modeling of exchange terms between phases (drag, evaporation) in a two-way coupled manner,

- mathematical and numerical analysis of the complete system including transport in phase and physical dimensions (3D),

- integration in moving mesh algorithms for geometric variations of the combustion chamber (piston, valves motions) and injector (needle motion), and

- applications in practical situations.

In the context of automotive injection, the modeling of the spray/gas turbulent interactions in engines is also an important issue, which is the subject of numerous recent studies (see [38, 100, 129, 121]). We would like to draw the reader's attention to the fact that the developments stated above and their first implementations and tests in a engine solver were conducted in a laminar-flow context. Developments on the turbulent aspects of the approach are to be described and validated with full details (see [34]).

The present work introduces the key features of Eulerian moment models with size polydispersion, starting with the main steps of its derivation to the first test cases in realistic direct injection conditions. Section 2 introduces the two-phase flow modeling framework adopted for model development whose aim is to achieve a robust method for the disperse-phase simulations. A statistical description based on a Williams-Boltzmann kinetic equation for the spray number density function (NDF) is considered. General considerations on Eulerian modeling are presented in section 3 and various moment-based approaches developed recently are introduced from section 3.2 to section 3.5.

The high-order moment method for size polydispersion, recently developed in view of automotive spray direct injection, is described in section 3.6 and section 3.7. The required adaptations for engine applications and the first test cases for spray injection systems are described in section 4. In particular, it is shown that such a numerical approach is as accurate and stable as Lagrangian particle methods for disperse spray conditions and can be operated 
in complex geometries such as those encountered for practical injection devices with large-scale variations in temporal and spatial dimensions. As stated above, the turbulence generation in the final high-velocity jet simulations will directly result in air motion due to two-way coupled spray/gas macroscopic interactions.

\section{Kinetic spray modeling}

The disperse-phase assumption for the liquid, applicable for dilute sprays with volume fraction $\Phi_{\mathrm{v}}<10^{-2}$, allows the use of the so-called kinetic spray model. This model is based on the Williams-Boltzmann equation [123, 124]. Although this represents a mesoscopic level of description, it is called kinetic by analogy with the mesoscopic kinetic theory of gases. Indeed, the assumptions made for the spray droplets [39] are similar to the ones made for the gas molecules when deriving the kinetic model. These assumptions are recalled here. The model describes the transport of the spray NDF and its evolution due to the physical phenomena such as evaporation, heat transfer or particle interactions applied to the spray, as external forces. The source terms responsible for the phase-space evolution of the NDF are analyzed here. Due to the disperse-phase assumption, the liquid influence on the gas phase is obtained through source terms in the gas-phase governing equations. The expressions for these source terms are provided in the framework of a kinetic spray description.

\subsection{Particle flow regimes}

Within the dilute limit assumption made here, the dynamics of a gas-droplet flow is ruled by two major effects. First, droplet-droplet collisions may occur in a two-phase flow, depending on the collision frequency. The flow can then be parameterized by the Knudsen number Kn

$$
\mathrm{Kn}=\frac{\tau_{c}}{\tau_{g}}
$$

with $\tau_{c}$ is the collision time of droplets and $\tau_{g}$ is the gas-phase characteristic time. This number represents the importance of droplet-droplet collisions relative to free transport, and is the equivalent of the Knudsen number defined from the gas kinetic theory [108]. Therefore, the flow behavior is characterized by Kn such as in the gas kinetic theory. The particle flow can be considered as a continuous flow as long as $\mathrm{Kn} \leq 1$. On the contrary, when $\mathrm{Kn}>0.1$, the rate of collisions is not significant enough to ensure that the flow is at equilibrium, i.e the velocity distribution function is Maxwellian, or at a state close to equilibrium.

Secondly, the drag is the leading phenomenon intervening in disperse phase flows which is the major actor allowing the distribution of droplets inside the combustion chamber. There are two main dimensionless numbers characterizing this behavior: the Stokes number St and the droplet Reynolds number $\operatorname{Re}_{p}$.

The Stokes number is expressed as:

$$
\mathrm{St}=\frac{\tau_{p}}{\tau_{g}}
$$

with $\tau_{p}$ is the dynamic time scale associated to a droplet of size in surface $S$, represents the response of a droplet to a change inside a gas flow. If the Stokes number is small enough $(\mathrm{St} \leq 1)$, the droplets will have nearly the same velocity as the gas. On the other hand, for large Stokes numbers, droplets barely feel the gas, so that their trajectory is hardly influenced by the gas. This issue will lead to the phenomenon called particle trajectory crossing PTC, which will be further discussed in 2.1.2. For an isolated droplet in a uniform gas, the droplet Reynolds number determines the net force of the gas on the droplet and expressed as:

$$
\operatorname{Re}_{d}=\frac{\rho_{g} S^{1 / 2}\left|\boldsymbol{u}_{g}-\boldsymbol{u}\right|}{\sqrt{\pi} \mu_{g}},
$$

where $\boldsymbol{u}$ is the droplet velocity. $\mathrm{Re}_{d}$ is important to characterize the flow regime around a droplet. A finite particle with a diameter greater than the smallest length scale (Kolmogorov scale) of the continuous flow can modulate the turbulence of the fluid in the vicinity of its interface through a wake effect. Otherwise if the particle diameter is smaller than the Kolmogorov length scale, it is a point particle and the fluid flow around it will not be influenced by the motion of the particle. Let us also remind that even if a particle has a smaller diameter than the smallest fluid length scale, its high inertia can lead a Stokes number greater than 1. All these regimes of particles are important in choosing the right disperse-phase models [4]. 


\subsubsection{Polydispersity}

As far as the disperse-phase topology is concerned, various sizes of droplets determine the flow regime. In fact, both phases and droplet-droplet interactions are governed by the size. Characteristic time scales of physical phenomena occurring in the disperse-phase are therefore driven by the size. For instance, the Stokes number St, depending on size through dynamic time scale $\tau_{p}$, previously given in Eq.(2) is one of the parameters influencing the velocity relaxation between the continuous phase and droplets. Yet one can also define other Stokes numbers in case where droplet evaporation and heating, determining the mass and heat exchanges between phases, are governing physics:

$$
\mathrm{St}_{v}=\frac{\tau_{v}(S)}{\tau_{g}}, \quad \mathrm{St}_{\Theta}=\frac{\tau_{\Theta}(S)}{\tau_{g}}
$$

with $\tau_{v}$ the evaporation time scale and $\tau_{\Theta}$ the thermal relaxation time scale, depending on size. The strength of polydispersity and the ways to quantify it depend on the application, but for internal combustion engine applications considered here, it has a crucial impact on the equivalence ratio, which conditions the combustions regime and the pollutant formation.

\subsubsection{Particle Trajectory Crossings (PTC)}

Particle trajectory crossings (PTC), are linked to the ability of droplets to have significantly different velocities in a same region of the flow. As the droplets tend to correlate their velocities at different rates according to their size in a given flow, it is natural to expect a major influence of size: while all the very small droplets have the gas velocity, small, medium and large droplets have a velocity depending on their size so that they do cross if their sizes are significantly different. This is referred to as hetero-PTC i.e. PTC at different sizes [24]. In addition, medium and large droplets may encounter crossings even for droplets of the same size, which is referred to as homo-PTC, PTC at same size [24]: this is due to the fact that medium and large droplets are defined to take a large time compared to that of the gas to correlate their velocities so these correlations weakly or never occur.

\subsection{Williams-Boltzmann kinetic equation: framework and derivation}

We present briefly the framework needed for the derivation of the Williams kinetic equation for a spray. For more details, the reader can see, for example, [123, 124].

\subsubsection{Basis assumptions}

Statistical modeling relies on ensemble (or statistical) averages. The goal is to reduce the information carried by the numerous particles to the only relevant one of ensemble behaviors. Each particle, denoted by the subscript $i$, is described by few degrees of freedom (DoF) that evolve in time, e.g. a minima its position $\boldsymbol{x}_{i}(t)$ and velocity $\boldsymbol{u}_{i}(t)$. So the particle system has $6 N$ DoFs in total and its state is exactly described by a point in a $6 N$-dimensional space called the $\Gamma$ phase space with $\Gamma=\mathbb{R}^{6 N}$. For classic particles encountering an external force $D_{\mathrm{r}}{ }^{e x t}$ per unit mass and a particle-particle interaction force $D_{\mathrm{r}_{i, j}}$ per unit mass that differs on the relative distance $\left|\boldsymbol{x}_{i}-\boldsymbol{x}_{j}\right|$, the equations of motion at the microscopic level read:

$$
\begin{aligned}
\mathrm{d}_{t} \boldsymbol{x}_{i}(t) & =\boldsymbol{u}_{i}(t), \\
\mathrm{d}_{t} \boldsymbol{u}_{i}(t) & =D_{\mathrm{r}}{ }^{e x t}(t)+\sum_{\substack{j=1 \\
j \neq i}}^{N} D_{\mathrm{r}_{i}, j}(t) .
\end{aligned}
$$

We want to avoid describing all the details of the system and, instead, to follow some of the quantities that emerge at the macroscopic scale. These quantities define a state at the macroscopic level and they are not as numerous as the micro-DoFs. So a macroscopic state can be reached thanks to many different underlying microscopic configurations [96]. We now focus only on these macroscopic variables: we compute them as ensemble averages, i.e. an average over many copies of the initial system, possibly evolving differently at the microscopic scale, but similarly at the macroscopic scale: this idea, due to Gibbs, allows to smooth out the undesired fluctuations from the temporal behavior of the macroscopic quantities. The number of ensembles to consider is large enough for the microscopic state points to be dense in $\Gamma$. We thus adopt a statistical point of view, introducing a multiple-particle 
joint distribution function $f^{N}\left(t, x_{1}, x_{2}, \cdots, x_{N}, u_{1}, u_{2}, \cdots, u_{N}\right)$. The evolution of an $N$-particle system is then given by the Liouville equation for the NDF in $6 N$ phase space:

$$
\partial_{t} f^{N}+\sum_{i}^{N} \boldsymbol{u}_{i} \cdot \partial_{x} f^{N}+\sum_{i=1}^{N}\left(D_{\mathrm{r}_{i}}^{e x t}+\sum_{\substack{j=1 \\ j \neq i}}^{N} D_{\mathrm{r}_{i, j}}\right) \cdot \partial_{\boldsymbol{u}_{i}} f^{N}=0
$$

which originates from the conservation of the number of points in $\Gamma$ and renders all the microscopic evolution equations of System (5a). In practice the dimension of $f^{N}$ is huge (it might be infinite) and one has to come up with a reduced (or contracted) description. Varying the number of particles retained in the state vector $\Gamma$, a reduced description is given by the classical BBGKY hierarchy (the initials are those of the authors who derived it independently: Bogoliubov, Born, Green, Kirkwood and Yvon) [17, 72]. It consists of transforming the Liouville equation into a chain of equations in which the first equation connects the evolution of one-particle PDF $f^{1}$ to the two-particle PDF $f^{2}$, the second equation connects $f^{2}$ to $f^{3}$ and so on. The system is an exact approach to the Liouville equation. It is therefore redundant but it is practical to build approximations by truncation of the chain when some particle correlations are neglected. The most celebrated example of the utility of the BBGKY hierarchy consists in closing the first equation of the hierarchy by assuming:

$$
f^{2}\left(t, \boldsymbol{x}_{1}, \boldsymbol{x}_{2}, \boldsymbol{u}_{1}, \boldsymbol{u}_{2}\right)=f^{1}\left(t, \boldsymbol{x}_{1}, \boldsymbol{u}_{1}\right) f^{1}\left(t, \boldsymbol{x}_{2}, \boldsymbol{u}_{2}\right)
$$

which means that the particles are totally uncorrelated. The BBGKY hierarchy is then entirely defined by the knowledge of a one-particle Probability Density Function (PDF) $f^{1}$ evolving in a 6-dimensional space called $\mu$ phase space according to the equation:

$$
\partial_{t} f^{1}+\boldsymbol{u}_{1} \cdot \partial_{x} f^{1}+D_{\mathrm{r}_{1}^{e x t}}^{e} \cdot \partial_{\boldsymbol{u}_{1}} f^{1}=-\int D_{\mathrm{r}_{1,2}} \cdot \partial_{\boldsymbol{u}_{1}} f^{1}\left(t, \boldsymbol{x}_{1}, \boldsymbol{u}_{1}\right) f^{1}\left(t, \boldsymbol{x}_{2}, \boldsymbol{u}_{2}\right) \mathrm{d} \boldsymbol{u}_{2} \mathrm{~d} \boldsymbol{x}_{2}
$$

The number density function (NDF) $f$, obtained by summing the one-particle PDF in order to describe $N$ indiscernible particles [120]: $f=\sum_{i}^{N} f^{i}$. Its phase space variables are $\boldsymbol{x}$ et $\boldsymbol{u}$. Similarly to Eq.(8) for the evolution of $f^{1}$, the Williams-Boltzmann equation is classically used to describe the transport of NDF function.

\subsubsection{Williams-Boltzmann equation}

The droplets are assumed to be spherical, and are characterized by their position, $\boldsymbol{x}$, their size $\phi$, their velocity $\boldsymbol{u}$ and their temperature $T$. The spray NDF depends on these variables and on time, the quantity $f^{\phi}(t, \boldsymbol{x}, \phi, \boldsymbol{u}, T) \mathrm{d} t$ $\mathrm{d} \boldsymbol{x} \mathrm{d} \phi \mathrm{d} \boldsymbol{u} \mathrm{d} T$ being the probable number of droplets with, at time $t$, a position in $[\boldsymbol{x}, \boldsymbol{x}+\mathrm{d} \boldsymbol{x}]$, a size in $[\phi, \phi+\mathrm{d} \phi]$, a velocity in $[\boldsymbol{u}, \boldsymbol{u}+\mathrm{d} \boldsymbol{u}]$ and a temperature in $[T, T+\mathrm{d} T]$. The particle size can be described either by the volume $V$, surface $S$ or radius $r$, with the following relation:

$$
f^{S} \mathrm{~d} S=f^{r} \mathrm{~d} r=f^{V} \mathrm{~d} V .
$$

It has been shown [62] that using the droplet surface as the variable for particle size is a good choice in the development of the multi-fluid model from the kinetic description. As shown in the next sections, it also proves to be a good choice for the development of the high order size moment method for the description of polydispersity. We therefore use surface $S$ as the size variable in the following development. The NDF $f$, without any superscript, will then be associated with the droplet surface.

The NDF of the spray follows a transport equation, the Williams-Boltzmann equation (WBE), first introduced in [123] and given as:

$$
\partial_{t} f+\nabla_{\boldsymbol{x}} \cdot(\boldsymbol{u} f)+\nabla_{\boldsymbol{u}} \cdot(\boldsymbol{F} f)-\partial_{S}(R f)+\partial_{T}(E f)=\Gamma+Q
$$

where

- $\partial_{t} f+\nabla_{\boldsymbol{x}} \cdot(\boldsymbol{u} f)$ represents the free transport of the spray,

- $\boldsymbol{F}=\mathrm{d}_{t}(\boldsymbol{u})$ is the acceleration applied on droplets per unit mass,

- $R=-\mathrm{d}_{t}(S)$ is the rate of change of the size $S$ of droplets, or evaporation term, 
- $E=\mathrm{d}_{t}(T)$ is the rate of change of droplet temperature due to heat transfer,

- $\Gamma$ is the rate of change of distribution function $f$ due to collisions,

- $Q$ is the rate of change of $f$ through particle formation by secondary break-up processes.

One can note the level of description of the disperse phase kinetic model. The influence of the gas on the spray is modeled through the terms $\boldsymbol{F}, R$ and $E$. These terms depend on the gas temperature, velocity and composition at the position of the droplet, and therefore are dependent on space and time.

The relationship presented in Eq. (10) is the general formulation of the Williams-Boltzmann equation that is typically presented in an introduction to spray modeling. Nevertheless, we will focus on the following terms of Eq. (10): free transport, external forces and the evaporation term. Isolating these effects will enable us to efficiently design numerical schemes for their resolution. Therefore, a simplified version of the Williams-Boltzmann equation to the basic kinetic equation satisfied by $f(t, \boldsymbol{x}, S, \boldsymbol{u})$, will be used throughout this work:

$$
\partial_{t} f+\nabla_{\boldsymbol{x}} \cdot(\boldsymbol{u} f)+\nabla_{\boldsymbol{x}} \cdot(\boldsymbol{F} f)-\partial_{S}(R f)=0
$$

It is important to remember that this simplified framework is not due to a limitation of the proposed models and it is a critical step in the design of efficient Eulerian methods for spray resolution. Contrary to initial impressions of over simplicity that one may harbor, these terms are the most challenging ones to solve when using high order moment methods. Indeed, recent publications point out intrinsic difficulties for the description of polydispersity through evaporation [41], and for the description of particle trajectory crossing (PTC) [40]. Models for collision and coalescence have been designed [64],[26] and prove to be very satisfactory even when combined with highorder moment methods for size [41] or velocity [45].

The next section discusses the possible choices for the resolution method for the spray model. A seemingly natural choice is the Lagrangian method where one tracks particles in the flow, considered as representing physical droplets. Other Lagrangian methods consist in tracking statistical particles in connection with Eq. (11). The widely used Lagrangian approach has proven its great efficiency to simulate complete spray dynamics without introducing any numerical diffusion $[31,93,2,98,89,103]$ except when coupling back to the gas phase is introduced. However, given the encountered limitations of Lagrangian methods in terms of physics and computing discussed below, an Eulerian spray description represents an interesting complementary tool, given its easier coupling with the gas phase, and its easier optimization through parallel computing, making it an interesting choice for high-performance computing (HPC) [10, 61]. 


\section{Eulerian moment methods for sprays}

The alternative to Lagrangian particle tracking is the resolution of Eulerian global quantities for the spray, such as number or mass density and momentum. Eulerian methods can be seen as moment methods derived from the kinetic equation Eq. (11). We propose to present here the main derivation strategies and resulting methods in the framework of a spray. We highlight in this presentation the key issues of Eulerian spray modeling in a combustion context related to the description of the velocity and size distributions. Indeed these two issues condition the ability of Eulerian methods to describe polydispersity and out-of-equilibrium velocity distributions, i.e., droplet trajectory crossing.

We present the main derivation strategies leading to Eulerian moment approaches, along with the associated assumptions. We distinguish between methods explicitly resolving the size distribution based on size-conditioned velocity equilibrium distribution functions and quadrature methods. Among the first class of methods, two families of models are isolated, relative to their treatment of the size variable. The first performs a discretization of the size phase space with finite-volume procedures. On the contrary, the second family relies on high order moment methods with continuous size reconstruction.

The developments and the spray computations presented in this section are done in a laminar flow context. Modeling spray-gas turbulent two-way coupled interactions using moment methods is a complex issue, which is currently being developed and tested in engine injection conditions and it is the subject of a separate paper [34] in the framework of RANS modeling. What is proposed in the following sections is to highlight the key points of each Eulerian moment method and its derivation, without introducing additional complexity related to turbulence modeling.

\subsection{Derivation strategies in a DNS context : Quadrature vs hydrodynamic velocity equi- librium conditioned in size}

The full resolution with finite volume of the kinetic equation, Eq. (11) (also referred to by O'Rourke as the full spray equation method [93]) is difficult given its cost related to the high number of phase space dimensions. Indeed in a $3 \mathrm{D}$ case, the phase space is of dimension seven ( 3 for space, 3 for velocity, 1 for size). Nevertheless, in many cases the knowledge of the full kinetic description of the spray is not needed, and it is sufficient to resolve the evolution of global quantities such as the NDF moments. For a given function $\psi(y)$, the $k^{\text {th }}$ order moment $m_{k}$ is defined by

$$
m_{k}=\int_{y} y^{k} \psi(y) \mathrm{d} y .
$$

Therefore, for the NDF we introduce the moment $\mathcal{M}$ of order $k$ in size, $(l, m, n)$ for each component of the velocity, respectively :

$$
\mathcal{M}_{k, l, m, n}=\int_{S} \int_{\boldsymbol{u}} f(t, \boldsymbol{x}, S, \boldsymbol{u}) S^{k} u_{x}^{l} u_{y}^{m} u_{z}^{n} \mathrm{~d} S \mathrm{~d} \boldsymbol{u}
$$

The evolution of the spray moments can be derived from the (WBE) equation Eq. (11), in the following way:

$$
\int_{S} \int_{\boldsymbol{u}} E q \cdot(11) S^{k} u_{x}^{l} u_{y}^{m} u_{z}^{n} \mathrm{~d} S \mathrm{~d} \boldsymbol{u},
$$

One has to notice that the moment equation derivation leads to a loss of information and that, without any peculiar assumption, the system of equations for moments is not closed, even if the kinetic model was. Indeed, some quantities can not be expressed in function of the set of moments resolved; for example with regard to velocity moments, the equation for the pth order moment (with $p=i+j+k$ ) introduces the moments of order $p+1$. Therefore taking one first order moment $\mathcal{M}_{0,0,1,0,0}$ introduces the second order moments $\mathcal{M}_{0,0,2,0,0}, \mathcal{M}_{0,0,1,1,0}$, $\mathcal{M}_{0,0,1,0,1}$ (see [118] and references therein). Assumptions have then to be done on the form of the NDF to close the moment evolution system. Therefore, there are two major strategies in the literature.

- For some of the spray Eulerian methods, the derivation of the moment system, along with its associated closures can be divided into two steps. First a form for the NDF in velocity and in temperature is presumed for each fixed size as follows:

$$
f(t, \boldsymbol{x}, \boldsymbol{u}, S)=n(t, \boldsymbol{x}, S) \phi_{\boldsymbol{u}}(t, \boldsymbol{x}, \boldsymbol{u}, S)
$$


where $\phi_{\boldsymbol{u}}$ is the presumed PDF in velocity, which is most of the time a hydrodynamic equilibrium from kinetic theory [118]. The variable $n$ is the number density of the spray. The velocity integration of (WBE) under the NDF Eq.(15) leads to the derivation of an intermediate closed system of conservation laws, the semi-kinetic system. The second step is devoted to the size-phase space treatment, there exist several methods introducing different types of system of conservation laws and closures.

- However, we can also find methods using quadrature-based approximations for the NDF introduced originally in $[85,126,76]$. One can write conservation equations for moments of WBE and use a quadrature formula to compute the unknown moments and source/forces integrals as a function of low order moments of the NDF. The difficulty relies in the quadrature, that is evaluating the abscissas and weights of the quadrature from the knowledge of the moments, for which there as very efficient algorithms where the distribution is monovariate, but becomes more involved when multi-variate distributions are investigated* . The Direct Quadrature Moment of Methods (DQMOM) quadrature method has been originally developed for cases where a multi-variate NDF function is required for WBE [76]. In order to evaluate its effectiveness in the context of evaporating polydisperse spray modeling, it has been also compared to the multi-fluid method in [41]. In this case, instead of transporting moments of the distribution, one transports all abscissas and weights of quadratures. A system of conservation laws is obtained, where source terms are derived from the WBE equation on a set of moments one wants to exactly resolve. Yet, in [41], it has been demonstrated that the DQMOM approach shows inaccuracies when it comes to predict droplet evaporations since as QMOM, it is hard for such an approach to recover the point-wise value at zero size from the knowledge of the moments related to the number of disappearing droplets. The more recently introduced EQMOM based on the ideas of more regular kernels than Dirac delta functions, but also relying on quadrature methods, is a promising method but there is still some work to be done in order to cover the entire moment space up to the boundary [128]. Moreover, DQMOM has been proven to be inaccurate for PTC events for which the CQMOM approach is rather preferred [127] even is it is weakly hyperbolic and can lead to artificial singularity formations [16]; once again Multi-Gaussian distribution reconstruction, on which EQMOM method has been built, can help solving these issues [15, 66, 14].

Even if the field of quadrature-based moment methods is quite active, since we will not be using this approach in order to treat properly the evaporation process, which has to be based on smooth reconstruction of the number density function, we will only concentrate on the exposition of the first class of methods. Before treating the polydispersion, we will propose a synthesis of polykinetic treatment of monodisperse sprays of droplets or particles based on kinetic theory approaches. Then, we will focus on to the Eulerian polydisperse models where the link with Eulerian polykinetic models will be also investigated.

\subsection{Eulerian polykinetic modeling through kinetic-Based Moment Methods (KBMM)}

The common aspect of Eulerian polykinetic models is the fundamental modeling of the velocity distribution function $\Psi_{\boldsymbol{u}}$ in Eq.(15). The basic mono-kinetic assumption for the velocity distribution that is a unique spray velocity

\footnotetext{
${ }^{*}$ The original QMOM approach consists in transporting and conserving a set of $2 N_{p}$ size moments $M=\left(M_{0}, \ldots, M_{2 N_{p}-1}\right)$ with $M_{k}(t, \boldsymbol{x})=\int_{\mathbb{R}^{+}} \xi^{k} f(t, \boldsymbol{x}, \xi) \mathrm{d} \xi$. The NDF function is presumed by the following expression: $f(t, \boldsymbol{x}, \xi)=\sum_{i=1}^{N_{p}} w_{i}(t, \boldsymbol{x}) \delta\left(\xi-\xi_{i}(t, \boldsymbol{x})\right)$ with $N_{p}$ is the number of peaks, $w_{i}$ the weights, and $\xi_{i}$ the abscissas. Thanks to the moment-inversion algorithm provided in [84], weights and abscissas are found from the relation $M_{k}=\sum_{i=1}^{N_{p}} w_{i} \xi_{i}^{k}, k \in 0,1, \ldots, 2 N_{p}-1$. To illustrate the QMOM method, let us work with a rather simplified WBE for the aerosols transported through the gas velocity $\boldsymbol{u}_{g}$
}

$$
\partial_{t} f+\nabla_{\boldsymbol{x}} \cdot\left(\boldsymbol{u}_{g} f\right)=\Lambda(t, \boldsymbol{x}, \xi) f(t, \boldsymbol{x}, \xi) .
$$

After the size integration, the evolution of the $k$ th order size moment is given by:

$$
\partial_{t} M_{k}+\nabla_{\boldsymbol{x}} \cdot\left(M_{k} \boldsymbol{u}_{g}\right)=\int_{\xi} \xi^{k} \Lambda(t, \boldsymbol{x}, \xi) f(t, \boldsymbol{x}, \xi) \mathrm{d} \xi
$$

The evolution of the NDF is therefore described by $2 N_{p}$ equations. Yet, The form of the function $\Lambda$ can be complex and yields unclosed moments. The strategy adopted in QMOM method is to first reconstruct quadrature points and then compute unclosed integral terms as well as non-conserved moments $\int_{\xi} \xi^{k} \Lambda(t, \boldsymbol{x}, \xi) f(t, \boldsymbol{x}, \xi) \mathrm{d} \xi=\sum_{i}^{N_{p}} \xi_{i}^{k} w_{i}(t, \boldsymbol{x}) \Lambda\left(t, \boldsymbol{x}, \xi_{i}(t, \boldsymbol{x})\right)$, as well as non-conserved moments $M_{\alpha}=$ $\sum_{i=0}^{N_{p}} w_{i} \xi_{i}^{\alpha}$ 
for all droplets at a given time and position, leading a dirac $\delta$-function $\Psi_{\boldsymbol{u}}=\delta_{\boldsymbol{u}}$, has been the starting point for some Eulerian polydisperse models, discussed in section (3.3). Yet the latter approximation is not true for twophase flows with inertial droplets in a gaseous carrier phase. In such flows the motion of droplets strongly depends on their inertia characterized by their Stokes number, based on the smallest time scale of the carrier flow which is, in case of a turbulent flow, the Kolmogorov scale or can be related to a strain rate as demonstrated by S. de Chaisemartin in his $\mathrm{PhD}$ [20]. For low Stokes numbers, the spray phase velocity is strongly correlated to the gas phase velocity and mono-kinetic velocity distribution is well adapted. Therefore, one can work with only velocity moments of zero and first order. Yet regimes with higher Stokes numbers St, droplets velocity are not well correlated locally with the gas velocity. This leads to an uncorrelated droplet motion either called the Random Uncorrelated Motion (RUM) [38] or the granular temperature [42]. The latter aspect drives the homo-PTC phenomenon (see part 2.1.2). It is important that the velocity distribution $\Psi_{\boldsymbol{u}}$ locally reproduces polykinetic distribution, that is locally various velocities for same sized droplets at time $t$ and location $\boldsymbol{x}$.

The complete hierarchy of Eulerian methods, aiming at capturing homo-PTC is discussed in detail in [66]. We can divide up the Eulerian polykinetic approaches of the literature into two categories:

- Algebraic-Closure-Based Moment Methods (ACBMM): A limited set of moments, usually up to second order moments, are chosen and transported. Since their transport involves higher order moments, these missing moments are computed from the knowledge of the lower order moments through equilibrium assumptions inspired from RANS turbulence modeling using explicit algebraic closures [107]. One example of this class of methods introduced in [79, 80] considers and transports a unique, scalar second order moment. Other second or third order moments are then computed from the knowledge of the transported moments to get the most accurate closure at a reduced cost. This type of approach has already reached the real application level $[122,78,100]$. However it has to face local realizability problems [105], i.e. the occurrence of moments not linked to a non-negative NDF, and the design of adapted numerics is not straightforward and has never been conducted since the mathematical structure of the underlying system of Eulerian equations is not clearly identified.

- Kinetic-Based Moment Methods (KBMM): The main idea of this type of approach is to consider a set of moments for which we can associate in a one-to-one correspondence a unique kinetic velocity distribution with a sufficient number of parameters to controlthe given set of moments. This presumed NDF must be non negative and allows to evaluate high order moments needed in the system of moments for transport. Closures have been proposed to control moments up to second order [81, 118], up to the third order (CQMOM [127]), and up to the fourth order (Multi-Gaussian [15, 66, 14]). Among the KBMM two categories emerge; the first is based on hydrodynamic equilibrium usually related to a given notional collision operator and the second is based on quadrature methods. The first category allows a well-defined mathematical structure and entropy inequality, whereas most of the time the second leads to weakly hyperbolic systems [16]. The main advantage with KBMM is the existence of dedicated numerical methods, which will guarantee the realizability and the stable behavior of the numerical schemes, either classical hyperbolic solvers [70, 113] or kinetic schemes [11, 20, 127].

\subsection{Kinetic based Eulerian simulation of polydisperse droplets}

We now focus on kinetic based Eulerian models able to describe both the polydispersity and the droplets evaporation. The idea is to briefly discuss about each of the several existing methods along their potential towards ICE applications.

The derivation of some of the Eulerian polydisperse models are done in two steps. The first step consists of obtaining the semi-kinetic equation system through the integration in velocity and temperature of WBE and the second step is the derivation of Eulerian equations through the integration in size of the semi-kinetic system. Yet for the sake of simplicity, these methods are originally derived, assuming a monokinetic assumption for the NDF function but higher velocity moments could be considered. This implies that there are locally unique velocity and temperature for all droplets. It is therefore useful to first introduce the semi-kinetic modeling concept based on this strong assumption before discussing about each of relevant polydisperse models.

We highlight here the main assumptions on the NDF and give its form under an equilibrium assumption with no dispersion for the velocity and the temperature distribution:

- [H1] For a given droplet size, at a given point $(t, \boldsymbol{x})$, there is only one characteristic averaged velocity $\boldsymbol{u}_{p}(t, \boldsymbol{x}, S)$. 
- $[\mathrm{H} 2]$ The dispersion in the distribution function around the mean velocity is zero in each direction, whatever the point $(t, \boldsymbol{x}, S)$.

- [H3] The dispersion in the distribution function around the mean temperature is zero in each direction, whatever the point $(t, \boldsymbol{x}, S)$.

It is equivalent to presume the following NDF conditioned by droplet size:

$$
n(t, \boldsymbol{x}, S)=f(t, \boldsymbol{x}, S) \delta\left(\boldsymbol{u}-\boldsymbol{u}_{p}(t, \boldsymbol{x}, S)\right) .
$$

From this approximation, we can derive a system of moment equations for the spray taking the moments in velocity of order 0 and 1 of WBE. This gives rise to the semi-kinetic system of equations:

$$
\begin{aligned}
& \partial_{t} n+\nabla_{\boldsymbol{x}} \cdot\left(n \boldsymbol{u}_{p}\right)=\partial_{S}\left(R_{p} n\right), \\
& \partial_{t}\left(n \boldsymbol{u}_{p}\right)+\nabla_{\boldsymbol{x}} \cdot\left(n \boldsymbol{u}_{p} \otimes \boldsymbol{u}_{p}\right)=\partial_{S}\left(R_{p} \boldsymbol{u}_{p} n\right)+\boldsymbol{F}_{p} n,
\end{aligned}
$$

with

$$
R_{p}=R\left(t, \boldsymbol{x}, \boldsymbol{u}_{p}, S\right), \quad \boldsymbol{F}_{p}=\boldsymbol{F}\left(t, \boldsymbol{x}, \boldsymbol{u}_{p}, S\right) .
$$

Let us recall that the semi-kinetic system, derived from the monokinetic assumption on the NDF, is weakly hyperbolic since no pressure like dispersion term arises in Eq.(17b). Therefore, Eulerian methods which will be derived through system (17) will follow this pressure-less formalism. This remark is particularly important since the pressure-less system is known to generate delta-shocks and do not capture the homo-PTC [20]. To overcome this difficulties, these polydisperse methods can be coupled to Eulerian polykinetic approaches [24] which are already presented in part 3.2 .

\subsection{Eulerian polydisperse approach with size sampling}

Let us start with the size sampling approach. The size sampling approach [63, 7] also referred to as Multi-Class method, or Lagrangian-in-size method [92], considers the NDF as sampled regarding the size variable, yielding $I$ classes of particles of same size, illustrated in Figure 3(a). The sampling approach is based on the following approximate NDF:

$$
n(t, \boldsymbol{x}, S)=\sum_{i}^{I} N_{i}(t, \boldsymbol{x}) \delta\left(\boldsymbol{u}-\boldsymbol{u}_{i}(t, \boldsymbol{x})\right) \delta\left(S-S_{i}(t, \boldsymbol{x})\right)
$$

where $N_{i}, S_{i}$ and $\boldsymbol{u}_{i}$ are respectively the droplet number density, size and velocity at the location $(t, \boldsymbol{x})$. With this form, droplets with the same size are gathered into classes where $i$ is the index of a class: the classes perform a sampling of the NDF over the whole phase space. The mass and momentum conservation equations for each class

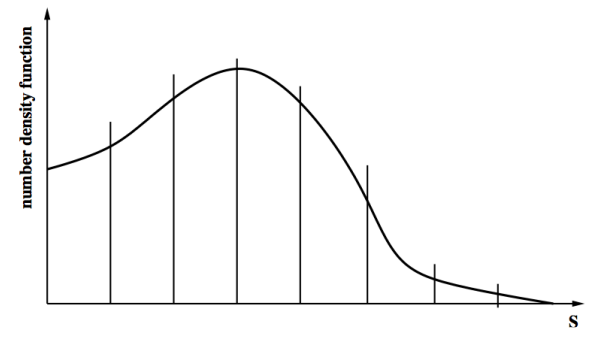

(a)

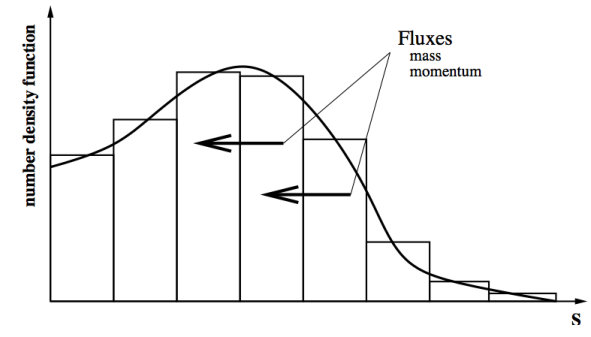

(b)

Figure 3. Size distribution with size sampling approach (a). Size distribution with MF method (b)

$i$ of the spray are obtained through the integration of WBE in size $S$ abd the velocity $\boldsymbol{u}$. The mass and momentum source terms are evaluated as they depend on the sampled droplet variables.

The Multi-Class approach can yield a satisfactory representation of the disperse phase size distribution, prevented that enough classes are considered and that they correspond to relevant points. Choosing a satisfactory 
sampling of a given distribution is a classical problem but here, it is coupled to the question of predicting the correct evolution of the spray, given such a distribution, which is a tough non-linear and implicit problem. So size samples are often chosen empirically. Moreover, as the approach is discretized regarding size with Dirac functions, the non-local transport cannot be accounted for properly: the size evolution due to sources (coalescence, break-up) is poorly rendered due to the fact that the samples have no particular reasons to match the new sizes. Conversely this technique is avoided when it comes to modeling discrete size-changing phenomena. Looking for the accurate evaporation, this method is not adapted for our problem.

\subsection{Eulerian Multi-fluid model}

The Eulerian Multi-Fluid model (MF), also referred to as 'sectional' method, was developed in [63] to account for the droplet size in a continuous and affordable manner. It is inspired from the seminal work in [112], furthered in $[106,47]$. But the origin and assumptions of the Multi-Fluid model have been precisely presented in [63]. Based on the mono-kinetic assumption, the original MF method is at first order in size for the evaporation [62]. Yet it has been extended to a second order in size for the evaporation through the work of $[62,25]$. Moreover, recent advances in [26] took into account size-velocity correlations for a rather precise description of hetero-PTC through two size moments. In this part, we will first focus on the derivation of the original MF, then providing some information on recent achievements and finally give a brief conclusion about the method.

\subsubsection{Original MF assumptions and the model derivation}

The original MF method is derived, adopting a mono-kinetic velocity and temperature distribution. Therefore this accounts of working with the same system given through Eq.(17). Yet, Eq.(17) has still a size phase-space $S$ dependence.

The Multi-Fluid model relies indeed on the choice of a discretization for the droplet size phase space:

$$
0=S_{0}<S_{1}<\cdots<S_{N_{s e c}}=\infty
$$

where $N_{\text {sec }}$ is the number of sections. The system of conservation laws is then averaged over each fixed size interval $\left[S_{k-1}, S_{k}\right.$ [, called section. The set of droplets in one section can be seen as a "fluid" for which conservation equation are written, the sections exchanging mass, momentum and energy. The principle of sectional discretization is shown in Figure 3(b) and can be seen as a finite volume method on the size dimension, continuous size-variation source terms (e.g. evaporation) resulting in fluxes at the edges of the size cells. In the following, let us recall the strategy to obtain a closed conservation equation system for each section through the integration in size of the semi-kinetic system (17).

The velocity and the temperature are supposed to be independent of size variable within each section. Therefore the notation $\boldsymbol{u}(t, \boldsymbol{x}, S)=\boldsymbol{u}^{(k)}(t, \boldsymbol{x})$ is chosen to designate the constant velocity distribution in section $k$. The validity of this assumptions is linked to the strength of polydispersity in each section, which is quantified in a section by comparing the smallest to the biggest Stokes numbers. If the dynamic Stokes number spectrum is too wide, the discretization must then be refined [20, 26, 27] or or size-velocity coupled high order moments should be used within each section to improve the accuracy [26, 122].

For the polydispersity treatment, in each section, the form of $n$ as a function of $S$ is presumed which allows to reduce the size distribution information in each section at $(t, \boldsymbol{x})$ to a set of moments of $S$.

In the original MF method [63], the One-size Moment (OSM) method which consists of decoupling the contribution in size $S$ from space-time $(t, \boldsymbol{x})$ dependence by the following way:

$$
n(t, \boldsymbol{x}, S)=\kappa^{(k)}(S) m_{3 / 2}^{(k)}(t, \boldsymbol{x})
$$

where $\kappa^{(k)}$ is a function of size $S$ and $m_{3 / 2}^{(k)}$ is the size moment corresponding to the spray mass density within the $k$ th section which is expressed as:

$$
m_{3 / 2}^{(k)}(t, \boldsymbol{x})=\int_{S_{k-1}}^{S_{k}} \frac{\rho_{p}}{6 \sqrt{\pi}} S^{3 / 2} n(t, \boldsymbol{x}, S) \mathrm{d} S=1
$$

which gives us, for the form $\kappa^{(k)}$ in one section:

$$
\int_{S_{k}-1}^{S_{k}} \kappa^{(k)}(S) \frac{\rho_{p}}{6 \sqrt{\pi}} S^{3 / 2} \mathrm{~d} S=1
$$


As mentioned in [63], $\kappa^{(k)}(S)$ is taken as constant in size distribution .

Let us now derive macroscopic MF equations. For the sake of simplicity, the spray temperature is assumed to be constant and uniform. From where, taking the integration in $\frac{\rho_{p}}{6 \sqrt{\pi}} S^{3 / 2}$ of the semi-kinetic system (17) within a section $\left[S_{k+1}, S_{k}\right.$ [ leads to the following system of conservation equations:

$$
\begin{aligned}
\partial_{t} m_{3 / 2}^{(k)}+\nabla_{\boldsymbol{x}} \cdot\left(m_{3 / 2}^{(k)} \boldsymbol{u}_{p}^{(k)}\right) & =-\left(E_{1}^{(k)}+E_{2}^{(k)}\right) m_{3 / 2}^{(k)}+E_{1}^{(k+1)} m_{3 / 2}^{(k+1)}, \\
\partial_{t}\left(m_{3 / 2}^{(k)} \boldsymbol{u}_{p}^{(k)}\right)+\nabla_{\boldsymbol{x}} \cdot\left(m_{3 / 2}^{(k)} \boldsymbol{u}_{p}^{(k)} \otimes \boldsymbol{u}_{p}^{(k)}\right) & =-\left(E_{1}^{(k)}+E_{2}^{(k)}\right) m_{3 / 2}^{(k)} \boldsymbol{u}_{p}^{(k)} \\
& +E_{1}^{(k+1)} m_{3 / 2}^{(k+1)} \boldsymbol{u}_{p}^{(k+1)}+m_{3 / 2}^{(k)} \boldsymbol{F}_{p}^{(k)},
\end{aligned}
$$

where we define, in the $k$ th section, the averaged velocity

$$
\boldsymbol{u}_{p}^{(k)}=\frac{1}{m_{3 / 2}^{(k)}} \int_{S_{k-1}}^{S_{k}} \frac{\rho_{p}}{6 \sqrt{\pi}} S^{3 / 2} \boldsymbol{u}_{p}(t, \boldsymbol{x}, S) n(t, \boldsymbol{x}, S) \mathrm{d} S,
$$

and the averaged drag term

$$
\boldsymbol{F}_{p}^{(k)}=\frac{1}{m_{3 / 2}^{(k)}} \int_{S_{k-1}}^{S_{k}} \frac{\rho_{p}}{6 \sqrt{\pi}} S^{3 / 2} \boldsymbol{F}_{p}(t, \boldsymbol{x}, S) n(t, \boldsymbol{x}, S) \mathrm{d} S .
$$

Expressions for the evaporation terms $E_{1}^{(k)}$ and $E_{2}^{(k)}$ are given as

$$
E_{1}^{(k)}=\frac{5 S_{(k-1)}^{3 / 2}}{2\left[S_{(k)}^{5 / 2}-S_{(k-1)}^{5 / 2}\right]} R_{p}\left(S_{(k-1)}\right)
$$

and

$$
E_{2}^{(k)}=\frac{5}{2\left[S_{(k)}^{5 / 2}-S_{(k-1)}^{5 / 2}\right]} \int_{S_{(k-1)}}^{S_{(k)}} \frac{3}{2} S^{1 / 2} R_{p}(S) \mathrm{d} S,
$$

\subsubsection{MF with two size moments per section}

Yet the OSM yields a lack of accuracy in terms of the size distribution and requires a great number of sections to counterbalance its drawback. An accurate method is therefore needed to capture polydispersity at the compromise of computational cost, but also ease of implementation and flexibility. Instead of increasing the number of sections, increasing the number of moments per section is a promising option. The works done in [62, 26] rely on solving two size moments in each section and the method is referred to as Two Size Moment (TSM) method. It consists in transporting moments corresponding to both the number $m_{0}^{(k)}$ and the mass $m_{3 / 2}^{(k)}$ instead of one unique moment per section. These moments are expressed as:

$$
\left(\begin{array}{c}
m_{0}^{(k)} \\
m_{3 / 2}^{(k)}
\end{array}\right)=\int_{S_{k}-1}^{S_{k}}\left(\begin{array}{c}
1 \\
\frac{\rho_{p}}{6 \sqrt{\pi}} S^{3 / 2}
\end{array}\right) n(t, \boldsymbol{x}, S) \mathrm{d} S
$$

The only realizability condition for a couple of size moments $\left(m_{0}^{(k)}, m_{3 / 2}^{(k)}\right)$ in a section is to be positive and to correspond to an average diameter that is in the size interval:

$$
\begin{aligned}
& \left(m_{0}^{(k)}>0 \text { and } m_{3 / 2}^{(k)}>0\right) \quad \text { or } \quad\left(m_{0}^{(k)}=m_{3 / 2}^{(k)}=0\right) \\
& \frac{\rho_{p}\left(S^{k-1}\right)^{3 / 2}}{6 \sqrt{\pi}} \leq \frac{m_{3 / 2}^{(k)}}{m_{0}^{(k)}} \leq \frac{\rho_{p}\left(S^{k}\right)^{3 / 2}}{6 \sqrt{\pi}}
\end{aligned}
$$

Respecting this realizability condition, there are several strategies in presuming an appropriate $n$. An exponential reconstruction called the exponential TSM (Exp-TSM) MF method, suggested in [30], was a first method, respecting the realizability condition. It has been proven to be well suited for evaporation, which requires mass flux information at the section boundary. The affine TSM (Aff-TSM) MF method is also based on a two-parameter approximation of the size distribution, through a positive affine function reconstruction, in each section. An early version was suggested in [62] and its more efficient extension has been recently proposed in [65]. 


\subsubsection{PTC with MF method}

As discussed before, droplets of different sizes can have different velocities due to the correlations between their sizes and velocities which yield to the hetero-PTC. Moreover, inertial droplets with the same size can have still different velocities, leading to the homo-PTC. Yet TSM method which captures the polydispersity resolution at a second order accuracy under a lower number of sections than OSM method, is not well adapted for the modeling of PTC. This is due to the fact that all droplets within a section share the same velocity and temperature. In [26], this lack of PTC accuracy has been addressed and MF method has been extended to capture the PTC.

- Hetero-PTC: Inspired from the work of Vié et al. [122] originally developed for high order moment methods (further detailed in section 3.7.6), the approach called Correlated Size-Velocity Two Size Moment (CSVTSM) method is designed to account efficiently for size-conditioned dynamics [26]. CSVTSM method, expected to be second order in size and velocity has been proven to suit well for coalescing cases. Yet additional to the two size moments transported in the original TSM MF method, two in 1D, four in 2D and six in 3D velocity moments per section are transported, having a significant impact on the CPU time.

- Homo-PTC: Inspired from the Kinetic Based Moment Methods (KBMM) [119, 66], MF extended and applied to moderate-inertia particle-laden flows in order to capture small scale PTC [118]. The velocity distribution in the NDF function is no longer taken as a Dirac function but based on an anisotropic Gaussian (AG) distribution given. MF method under AG distribution is shown to be well-suited to account for homo-PTC $[28,26]$. The method is promising for moderately dense polydisperse two-phase flows, to treat the portion of coalescing droplets that has a significant enough inertia.

\subsubsection{Cost and precision of the method}

The main drawback of the multi-fluid model is the cost associated to the resolution of the $N_{s}$ systems of conservation laws needed to obtain the evolution of the droplet mass in the sections. Indeed it has been shown [20] that the multi-fluid model is a first-order method in size, with potential extension to second order [62],[26, 24], and thus a high number of sections, with a minimum of ten sections, is needed to obtain a precise description of the evaporation process. This raises a twofold problem, in terms of theory and of practical computation. First, in terms of applied mathematics, one may want to overcome this low-order limitation and thus design a high-order method in size. Moreover, as shown in Fig. (4), the results are very different whether one or ten sections are considered. These results show that a minimum number of sections is required to get an acceptable accuracy. Although this number varies according to the physical case studied, ten sections can be considered the minimum for acceptable description of polydispersity. But then the computational cost is multiplied by ten, and is too prohibitive in an industrial context.

We provide an illustration in a configuration emphasizing this shortcoming. The Taylor-Green configuration for the gas phase is used, with a motionless droplet cloud, where the maximum droplet diameter is $d_{0}=15 \mu \mathrm{m}$. This value for the maximum droplet diameter is chosen in order for the Stokes number of the largest droplets $\mathrm{St}=$ 0.03 to be lower than the critical Stokes number $\mathrm{St}_{c}=1 / 8 \pi$. Results are provided for different discretizations of the size phase space: 10 and 40 sections. The sprays are evaporating, with an evaporation coefficient $R_{S}=0.27$. Indeed, Fig. (5)(a) exhibits the evolution of the mean particle surface of the total distribution through evaporation, in the analytical case, and for the multi-fluid model with 10 and 40 sections. Although the multi-fluid model with 40 sections still overestimates the mean particle surface, it is closer to the analytical solution than with 10 sections, despite an overall similarity of these two solutions. The explanation for this surprising similarity is given in Fig. (6) displaying the resulting mass of particles such as $S^{(j)}<0.5$ (the first 5 sections in the 10 section case, and the first 20 sections in the 40 section case) first, and $S^{(j)}>0.5$ (the remaining sections). These results are shown for $t=2$, when, given the value of the evaporation coefficient, there must be almost no particle such as $S^{(j)}>0.5$. If this is the case for the 40 section model, there is still a considerable mass of these droplets in the 10 section case. This resulting mass is due to diffusion in size phase space. The second conclusion to be drawn from Fig. (6) is that the dynamics of particles such as $S^{(j)}<0.5$ have the expected behavior, i.e., they are dragged more by the gas in the 40 section than in the 10 section case. These observations illustrate the need for a method able to reach a good accuracy in description of polydispersity with a reduced number of sections.

\subsubsection{Conclusion on MF method}

So far in this part, one has discussed the capability of the MF model to capture the physics of polydisperse evaporating sprays. However, even though this approach has been extended to be more accurate by Laurent et al. [65] 
and [26] for evaporating sprays, the necessity to discretize the size phase space can be a stumbling block. In that context, the Eulerian Multi-Size Moment (EMSM) method developed in [57, 83, 60] provides to increase the polydispersity accuracy within each section while diminishing the number of sections. Yet it has been proven in [60], a very good accuracy under a shorter CPU time then MF method is obtained even with the use of only one section. More details on EMSM method will be given in section 3.7.
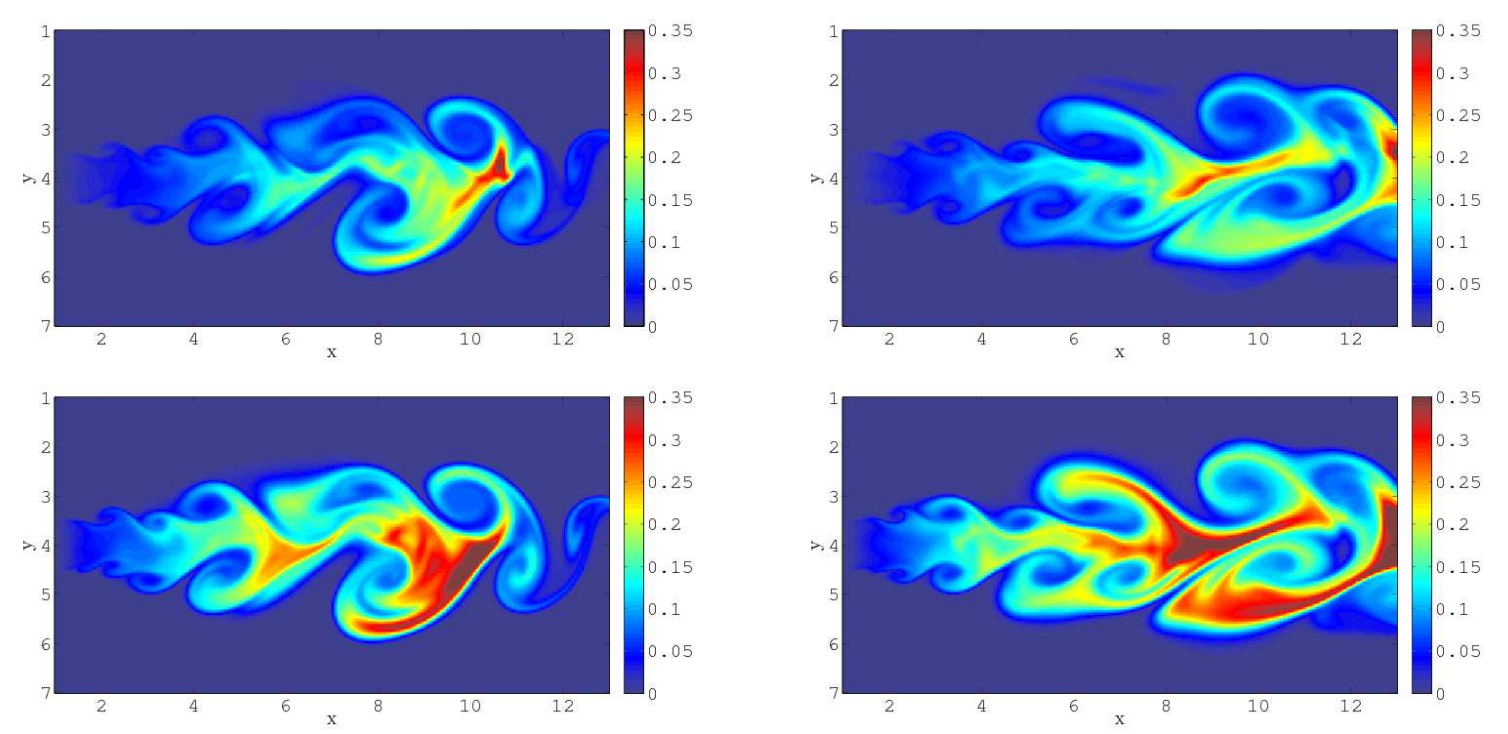

Figure 4. Comparison of the gas-phase fuel mass fraction at times $t=15$ (left) and $t=20$ (right). (Top) Multi-fluid model with one section. (Bottom) Multi-fluid model with ten sections.

\subsection{High order moment methods based on the Maximum entropy NDF reconstruction}

A promising alternative to quadrature-based moment methods is to reconstruct, among the infinity of solutions in the moment space, the unique NDF function, which maximizes the Shannon Entropy (ME) through its low order moments [86, 111], see Figure 5(b). Yet there are also other NDF reconstruction choices, such as the ones used in the description of aerosol Extended QMOM (EQMOM): a sum of beta PDF or gamma PDF, with the possibility to degenerate on quadratures, but sometimes loosing the highest moment in the reconstruction [128], but the full extent of the moment space is harder to reach with such methods.

ME reconstruction technique and its associated numerical strategy for the accurate evaluation of evaporation dynamics has been proved to be very promising for applications in relations with polydisperse sprays [83]. Therefore, inspired from the basic assumptions of MF method [63], the Eulerian Multi Size Moment (EMSM) method has been developed in $[57,83,60]$. The ability of spray simulations under the unstructured mesh motion through EMSM has been successfully assessed in [59]. It has been shown in [60] that EMSM can reach comparable levels of accuracy, with a reasonable space discretization, with reference to a Lagrangian simulation, while leading to a much lower level of computational cost compared to the standard MF approach. Let us also mention that a more recent work called Coupled Size-Velocity Moment (CSVM) method has been developed, in [119], as an extension model of the EMSM, taking into account size-velocity correlations in the spray. In [119], a tabulation technique has been also developed to decrease significantly the CPU time associated to size moment reconstruction.

\subsection{Eulerian Multi-Size Moment (EMSM) method}

It has been previously shown that, although the MF method offers an accurate resolution for the evaporating polydisperse spray, the necessity to discretize the size phase space, making use of several sections, requires to transport a system of governing equations for each section, thus increasing the CPU time. Let us recall that the computational time can be decreased through the use of a two-size moment MF method, while maintaining a good accuracy on the size distribution. Yet still more than one section is required. In [83], a four-size moment MF method has been developed, leading a very good accuracy both in case of only two and even one size section. 


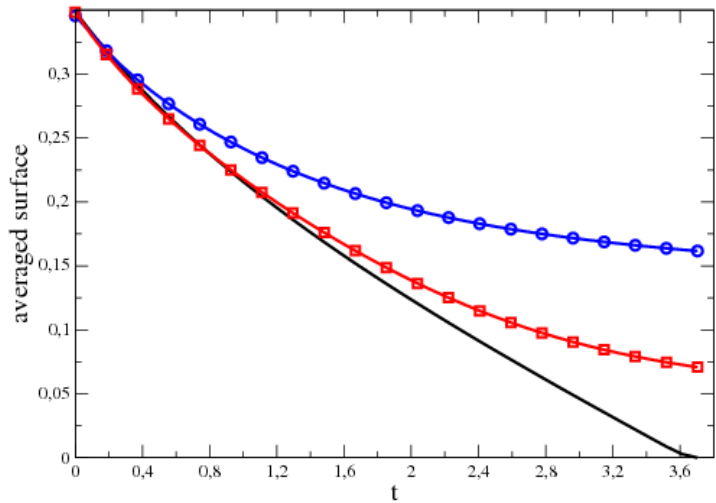

(a)

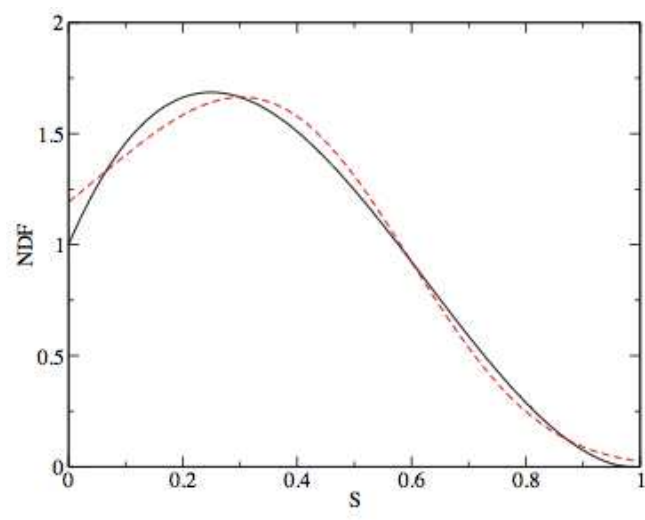

(b)

Figure 5. (a) Evolution of the mean particle size through evaporation with a $d^{2}$ law, and comparison with the analytical solution. Black curve: analytical solution. Dashed blue curve: high-order moment method. Blue curve with circles: multi-fluid model with 10 sections. Red curve with squares: multi-fluid model with 40 sections.(b) Reconstruction of the size distribution through entropy maximisation (red dashed line), the real size distribution (black solid line).

Since one section is enough to capture accurately the polydisperse evaporating spray, this one-section technique has been adopted in developing the high order moment method, called Eulerian Multi-Size Moment (EMSM) method as already presented to be accurate in part 3.6, while providing lower CPU time. In the reminder of this section, let us go into details of the EMSM method with four-size moments ${ }^{\dagger}$. Firstly, its derivation strategy will be given. Then the bottleneck points in working with several size moments along with the associated realizability condition will be point out. Afterwards, we will discuss about dedicated numerical schemes developed in the literature and recent fruitful realizations. Then, one will briefly discuss about the recent work initiated from the basis of EMSM to capture the hetero-PTC. Finally, its implementation in the industrial code IFP-C3D as well as required developments towards ICE applications will be discussed.

\subsubsection{Derivation of EMSM}

As was the case for MF method, the EMSM method is also based on a mono-kinetic assumption for both the temperature and the velocity distribution. Yet deriving a size moment system at macroscopic (Eulerian) level is rather different in EMSM compared to MF since the size distribution function $n$ is kept smooth and following assumptions are done:

$$
\boldsymbol{u}_{p}(t, \boldsymbol{x}, S)=\boldsymbol{u}_{p}(t, \boldsymbol{x})
$$

For the sake of simplicity, let us now take $R$ in $\mathrm{WBE}$ as a constant, so that $R=R_{p}$. One first takes the moments of Eq.(17a) in size of order 0 up to 3. Then one takes the size moment of order one of Eq.(17b). Then the governing equations are given as:

$$
\begin{aligned}
& \partial_{t} m_{0}+\nabla_{\boldsymbol{x}}\left(m_{0} \boldsymbol{u}_{p}\right)=-R_{p} n(S=0), \\
& \partial_{t} m_{1}+\nabla_{\boldsymbol{x}}\left(m_{1} \boldsymbol{u}_{p}\right)=-m_{0} R_{p}, \\
& \partial_{t} m_{2}+\nabla_{\boldsymbol{x}}\left(m_{2} \boldsymbol{u}_{p}\right)=-2 m_{1} R_{p}, \\
& \partial_{t} m_{3}+\nabla_{\boldsymbol{x}}\left(m_{3} \boldsymbol{u}_{p}\right)=-3 m_{2} R_{p}, \\
& \partial_{t}\left(m_{1} \boldsymbol{u}_{p}\right)+\nabla_{\boldsymbol{x}}\left(m_{1} \boldsymbol{u}_{p}^{2}\right)=-R m_{0} \boldsymbol{u}_{p}+\frac{18 \pi}{\rho} \mu_{g} m_{0}\left(\boldsymbol{u}_{g}-\boldsymbol{u}_{p}\right) .
\end{aligned}
$$

with

$$
m_{k}=\int_{0}^{S_{\max }} S^{k} n(t, \boldsymbol{x}, S) \mathrm{d} S,
$$

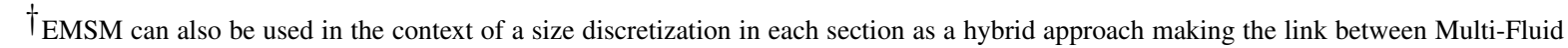
and high order moment method [122] 

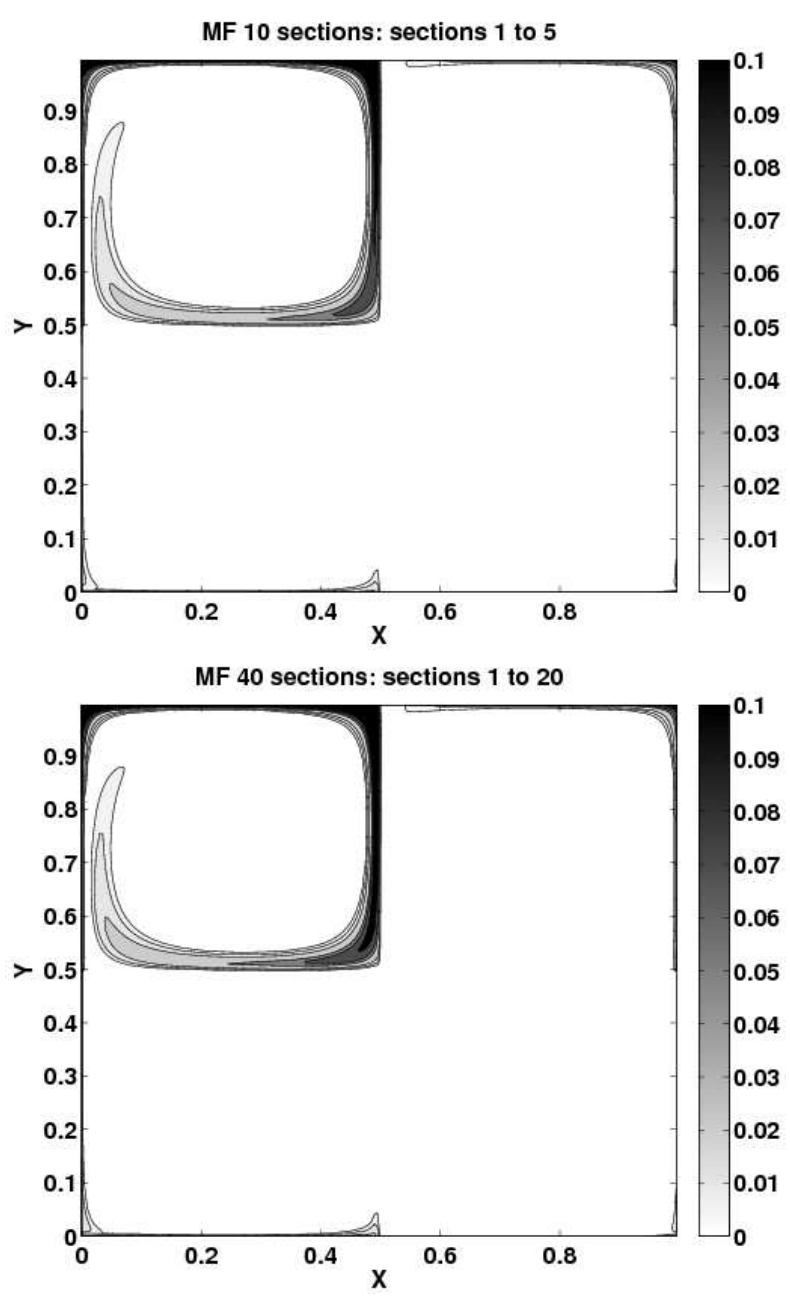
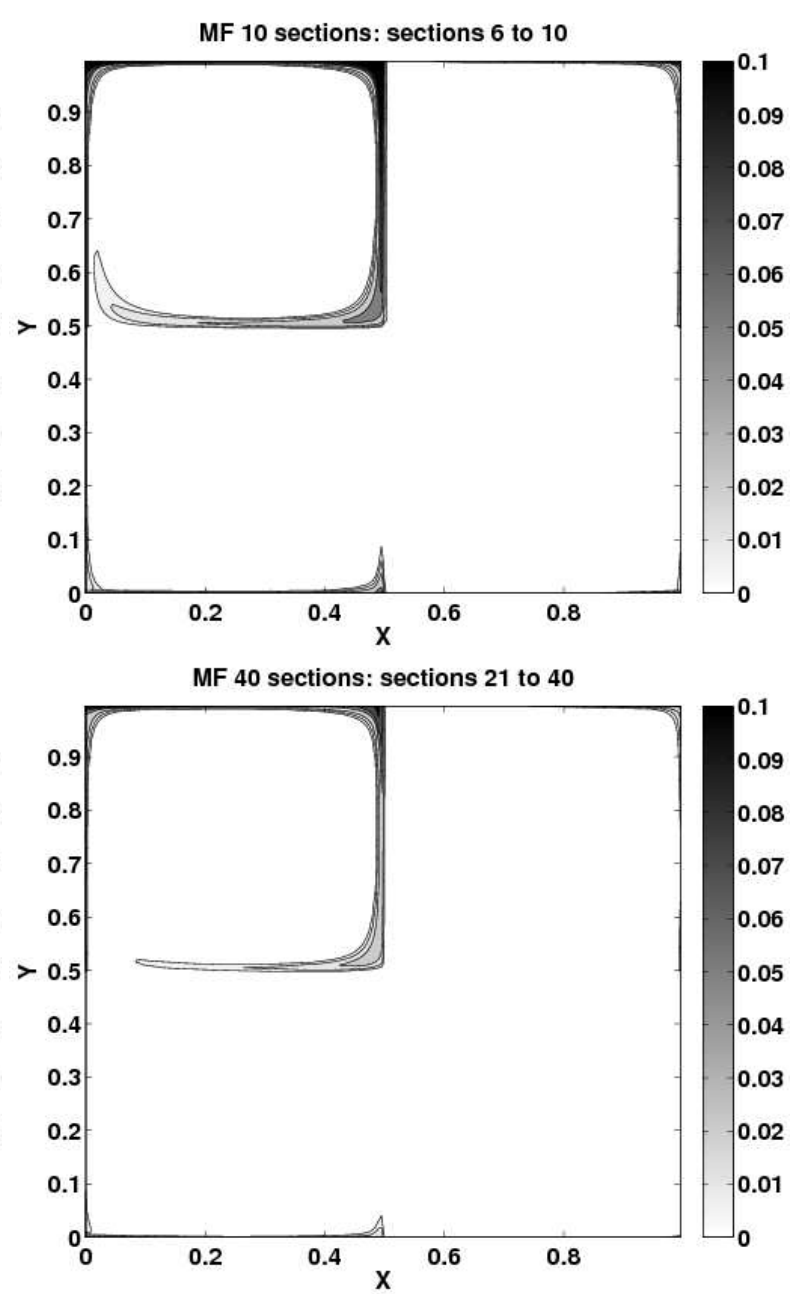

Figure 6. Comparison of the final mass in the two halves of the size phase space between the 10 section discretization and the 40 section discretization, in the evaporating case. (Top) 10 section discretization. (Left) mass in the first half of the size phase space (sections 1 to 5). (Right) mass in the second half of the size phase space (sections 6 to 10). (Bottom) 40 section discretization. (Left) mass in the first half of the size phase space (sections 1 to 20). (Right) mass in the second half of the size phase space (sections 21 to 40). 
and the unclosed term $n(S=0)$ represents the number of disappearing droplets due to evaporation. $S_{\max }$ is the maximum size of the distribution. The system (32) has been investigated in [57, 83, 60] from both modeling and numerical point of view. The realizability issue is raised as the moment space in which the moment vector lives is a convex subspace of $\left(\mathbb{R}_{+}\right)^{4}$ but with a quite complex shape, as explained in part 3.7.2. Numerical methods (e.g. transport schemes) must be carefully developed to avoid approximations of the moment vector that would be out of the moment space, resulting in failure of the simulation.

Phenomena involved in (32) (i.e, evaporation and convection) can be decoupled through an operator splitting approach [70]. Therefore, the subsystem that represents the evolution due to the evaporation and Stokes drag is given as:

$$
\begin{aligned}
& \partial_{t} m_{0}=-R_{p} n(S=0), \\
& \partial_{t} m_{1}=-m_{0} R_{p}, \\
& \partial_{t} m_{2}=-2 m_{1} R_{p}, \\
& \partial_{t} m_{3}=-3 m_{2} R_{p}, \\
& \partial_{t}\left(m_{1} \boldsymbol{u}_{p}\right)=-R_{p} m_{0} \boldsymbol{u}_{p}+\frac{18 \pi}{\rho} \mu_{g} m_{0}\left(\boldsymbol{u}_{g}-\boldsymbol{u}_{p}\right),
\end{aligned}
$$

whereas the one for the convection is expressed as:

$$
\begin{aligned}
& \partial_{t} m_{0}+\nabla_{\boldsymbol{x}}\left(m_{0} \boldsymbol{u}_{p}\right)=0, \\
& \partial_{t} m_{1}+\nabla_{\boldsymbol{x}}\left(m_{1} \boldsymbol{u}_{p}\right)=0, \\
& \partial_{t} m_{2}+\nabla_{\boldsymbol{x}}\left(m_{2} \boldsymbol{u}_{p}\right)=0, \\
& \partial_{t} m_{3}+\nabla_{\boldsymbol{x}}\left(m_{3} \boldsymbol{u}_{p}\right)=0, \\
& \partial_{t}\left(m_{1} \boldsymbol{u}_{p}\right)+\nabla_{\boldsymbol{x}}\left(m_{1} \boldsymbol{u}_{p}^{2}\right)=0 .
\end{aligned}
$$

For the numerical resolution of both (34) and (35), the main issue is to keep the integrity of moment set at anytime of the computation. An appropriate closure for the term $n(S=0)$ in Eq.(34a) and an accurate evolution of moments $m_{k}$ through (34a)-(34d) due to evaporation require particular numerical strategy which will be detailed in part 3.7.3. The resolution of the pressure-less system (35) will be explained in section 3.7.4.

\subsubsection{Moment space issue}

The major challenge for numerical methods designed for evaporation and transport is to keep the integrity of the moment vector $\boldsymbol{m}$, i.e to ensure that $\boldsymbol{m}=\left(m_{0}, m_{1}, m_{2}, m_{3}\right)^{t}$ belongs to the moment space at any time of the resolution process. Yet, even if the moment space $\mathbb{M}^{3}\left(0, S_{\max }\right)$ where lies the size moment vector $\boldsymbol{m}$ is convex, it has a complex geometry in the semi-open space $\left(\mathbb{R}^{+}\right)^{3}$ [83]. A simpler space can be determined by using the canonical moments [23]. The geometry of the space of the canonical moments vectors is much more simpler than the one of the moments since it is then the cube $[0,1]^{3}$. The canonical moments are then very useful in order to check the belonging to the moment space and design numerical schemes for system (34) and (35).

Let us recall that in case of the evaporation, one faces with the Hausdorff finite moment problem for the moments $\boldsymbol{m}$ which is finding a positive real valued function $\tilde{n}$ defined on $\left[0, S_{\text {max }}\right]$ from its moment set $\boldsymbol{m}$. This is a bottleneck problem for the accurate evaluation of the evaporative flux $n(S=0)$ in Eq.(34a). In [83], the solution for $\tilde{n}$ is based on the NDF reconstruction, using entropy maximisation of the moment set $\boldsymbol{m}$ which allows to evaluate the evaporative flux and droplet size evolution through a kinetic based numerical scheme for the evaporation [83].

\subsubsection{Evaporation scheme}

In [83], it has been shown that the numerical solution of the equation system (34a)-(34d) within a time step $\Delta t$ can be given as:

$$
\exp \left(\Delta t R_{p} \boldsymbol{A}\right) \boldsymbol{m}(\Delta t)=\boldsymbol{m}(0)-\Phi^{-}(\Delta t)
$$


where the flux at zero size $\Phi^{-}$is expressed as:,

$$
\Phi^{-}(\Delta t)=\int_{0}^{\Delta t R_{p}} \tilde{n}(\boldsymbol{m}, \beta)\left[\begin{array}{c}
1 \\
\beta \\
\vdots \\
\beta^{N}
\end{array}\right] \mathrm{d} \beta
$$

and the translation nilpotent matrix $\boldsymbol{A}$ is given as follows:

$$
\boldsymbol{A}=\left[\begin{array}{cccccc}
0 & & & & 0 \\
1 & 0 & & & \\
& 2 & \ddots & & \\
& & \ddots & \ddots & \\
0 & & & N & 0
\end{array}\right]
$$

and has the following property

$$
\forall t, \beta \quad \exp (\boldsymbol{A} t)\left(\begin{array}{c}
1 \\
\beta \\
\vdots \\
\beta^{N}
\end{array}\right)=\left(\begin{array}{c}
1 \\
\beta+t \\
\vdots \\
(\beta+t)^{N}
\end{array}\right)
$$

Starting from the Maximum entropy reconstruction of the size distribution $\tilde{n}$ (the technique introduced in 3.6), the evaluation of the evaporation process through Eq.(36) is made in two steps.

First, the disappearance flux $\Phi^{-}$at zero size is evaluated, and corresponds to the part of the moment that will disappear during a time step $\Delta t$. The moments are then corrected as:

$$
\hat{\boldsymbol{m}}=\boldsymbol{m}(0)-\Phi^{-}(\Delta t)
$$

where $\left.\hat{\boldsymbol{m}} \in \mathbb{M}^{N}\left(\Delta t R_{p}, S_{\max }\right)\right)$. Then, as far as $\hat{\boldsymbol{m}}$ is computed, one needs to compute the remaining contribution

$$
\exp \left(\Delta t R_{p} \boldsymbol{A}\right) \boldsymbol{m}(\Delta t)=\hat{\boldsymbol{m}} \text {. }
$$

This accounts for evolving the size distribution by means of the translation of its moments in size phase space [83]. Recalling that for any $\hat{\boldsymbol{m}}$, there exists one unique lower principal representation technique of the vector of moments by using weights $\left(w_{i}\right)_{i \in[1, n]}$ and abscissas $\left.\left(\hat{S}_{i}\right)_{i \in[1, n]} \in\right] \Delta t R_{p}, S_{\text {max }}[$ Therefore the corrected moments are then expressed, thanks to the quadrature points $i$, as

$$
\hat{\boldsymbol{m}}=\sum_{i=1}^{n} w_{i}\left(\begin{array}{c}
1 \\
\hat{S}_{i} \\
\hat{S}_{i}^{2} \\
\vdots \\
\hat{S}_{i}^{N}
\end{array}\right)
$$


where weights $\left(w_{i}\right)$ and abscissas $\hat{S}_{i}^{k}$ are computed through the Product Difference (PD) algorithm, for $n=2$, given in [84]. Thanks to the structure of matrix $\boldsymbol{A}$ given in Eq.(38), $\boldsymbol{m}$ is computed through quadrature points associated to $\hat{\boldsymbol{m}}$ as

$$
\boldsymbol{m}(\Delta t)=\sum_{i=1}^{n} w_{i}\left(\begin{array}{c}
1 \\
\hat{S}_{i}-R \Delta t \\
\left(\hat{S}_{i}-R_{p} \Delta t\right)^{2} \\
\vdots \\
\left(\hat{S}_{i}-R_{p} \Delta t\right)^{N}
\end{array}\right) .
$$

\subsubsection{Convection scheme}

In this part, we briefly discuss about the numerical scheme used to discretize the equations of system (35). Because of the conservative form of system (35), the finite-volume method [70] is a natural candidate for its discretization.

Usually, high order finite volume methods use some non-constant reconstructions of the variables to evaluate the fluxes between the cells. But, the properties of the scheme are conditioned by the expression of the fluxes. Two difficulties arise here. The first one concerns the way to do the reconstruction of the moments in order to keep the integrity of moment set. It has been seen that for a high order in space scheme, an independent reconstruction of each moment does not insure that the moment spaceis preserved [125, 85]. A second difficulty concerns the computation of the fluxes from the reconstructed quantities. If an approximate time solver is used (Explicit Euler, Runge-Kutta), the fluxes computation will introduce errors for non constant reconstructions, and the preservation of the moment space would not be guaranteed any more.

In [60], a second order scheme through piecewise linear reconstructions of conserved quantities within cells are considered. In order to render realizable the scheme, the canonical moments, which are proven to be transported quantities by system (35) and to satisfy a maximum principle are used for the reconstruction. For the time integration, a kinetic-based numerical scheme, using the ideas developed by Bouchut [11], has been developed [60]. For the latter, the fluxes are accurately evaluated, thanks to the exact resolution of the kinetic equation.

\subsubsection{Academic realizations through EMSM method}

Through academic test-cases, the robustness, the accuracy and computational efficiency of the EMSM method along with its numerical schemes and algorithms have been tested within 2D test-cases [60, 57]. Firstly, its is implemented in a Eulerian solver dedicated to spray simulations called MUSES3D [20, 82], developed at EM2C Laboratory carried out considering a stationary gas-phase through Taylor Green Vortices. Then, the free jet simulations are conducted with an academic solver, coupling the ASPHODELE solver, developed at CORIA by Julien Reveillon and collaborators [99, 97], with MUSES3D. For the latter case, the gas is therefore not frozen.

The evaporating polydisperse spray described through EMSM method has been successfully validated from both qualitative and quantitative point of view, comparing it to MF method (see figure 7) for each simulation.

\subsubsection{Recent extension of EMSM method to capture hetero-PTC: CSVM method}

Despite its ability to accurately capture the size evolution of polydisperse evaporating droplets, the EMSM method lacks of accuracy to capture size-velocity correlations of droplets. This is due to the assumption of unique velocity for all droplets sharing the same location at a given time. Yet the work conducted in [122] has recently addressed this issue and a new method called Coupled Size-Velocity Moment (CSVM) method has been developed. In that case the NDF function is taken as the one given through Eq.(16). The profile for the velocity $\boldsymbol{u}_{p}$ has been presumed in terms of the size such that droplets with a zero size share the velocity with the gas-phase. Therefore, the numerical scheme originally developed for EMSM method has been extended since one needs to transport one more moment per spatial dimension due to the new size-velociy correlation assumption done at the kinetic level. Moreover, some further numerical efforts are required since the size-velocity correlation issue can yield distributions very close to the boundary of moment space. In such case, in order to make converge Maximum entropy algorithm with a reasonable CPU time and increase the accuracy of the method, the following numerical development has been achieved in [122]: 

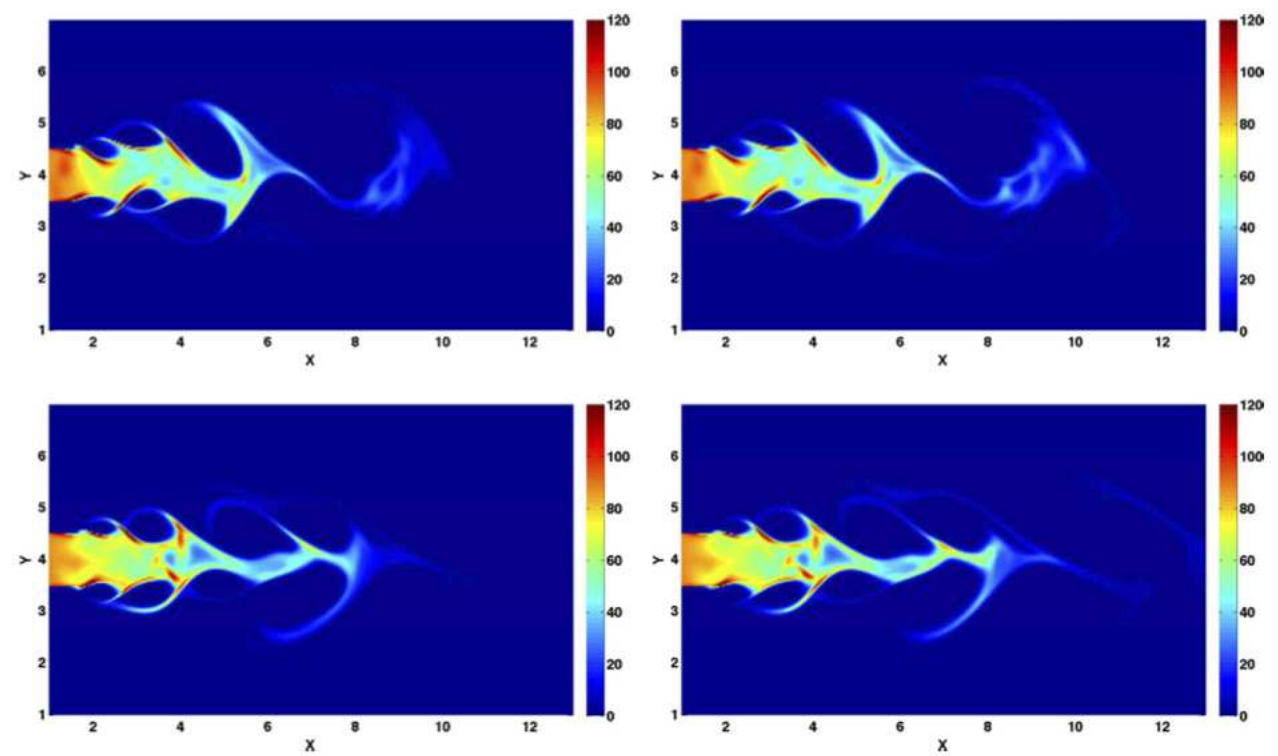

Figure 7. Total mass density of the polydisperse evaporating spray. (Top) Results at time $t=15$. (Bottom) Results at time $t=20$. (Left) EMSM model. (Right) Multi-fluid model with ten sections.

- To reduce the number of iterations needed for the Newton solver to converge, a tabulation method to calculate the NDF from its moments and associated interpolation methods have been investigated. It has been shown that the tabulation of the initial guess lowers significantly the number of Newton iterations needed, the fastest interpolation method being the more accurate third order polynomial reconstruction for the initial guess.

- To increase the accuracy of the reconstruction, an adaptive support for the Gauss-Legendre method has been implemented, that is, quadrature points are used only where the distribution is not too close to zero.

- At moment space boundaries, the distribution function represents the sum of diracs which involves a lower number of parameters less than the number of moments $N$. So the idea is that there is a transition zone in which one goes from a situation where $N$ parameters ( $\mathrm{N}$ moments) are needed (the interior of the moment space), to a situation where less parameters are needed (the frontier). This implies the use of less than $N$ moments at borders of moment space. In this case, the number of moments used is defined such that one has the same level of accuracy as the interior of moment space obtained through $N$ moments.

Through academic studies, the CSVM method along with its numerical tools have been proved to be more efficient than the original EMSM method in capturing the segregation inside the polydisperse spray with inertial droplets characterized by large Stokes number [122]. This is the direct consequence of capturing the hetero-PTC through CSVM method. 


\section{Implementation and tests in an engine solver}

In the context of internal combustion engine applications such as the direct injection of the liquid fuel, it is important to model the gas-phase entrainment by the polydisperse spray in the chamber under stiff injection conditions, while taking into account the presence of moving engine parts. In contrast to previous EMSM-related work [83, 60] where calculations were made on fixed meshes, the realistic configurations for injection in combustion engines with a moving piston require computations on moving meshes. The most common technique to cope with this new constraint is the Arbitrary Lagrange Euler (ALE) formalism, which was originally introduced in the context of single-velocity fluid flows [53]. Based on the same philosophy, the necessary numerical adaptations of the EMSM approach to ALE formalism has been recently developed and given access to simulations where two fluids, the compressible gas and the polydisperse spray coexist [35]. Moreover, based on the splitting strategy provided by ALE method, a new robust and accurate two-way coupled EMSM method has been developed, describing strong mass and momentum interactions between the compressible gas and evaporating polydisperse spray [35, 58]. All these developments for EMSM method have been integrated in the industrial CFD code IFP-C3D dedicated to compressible and reactive flow simulations under moving geometries. In the reminder of this chapter, first, the core structure of the ALE formalism with adaptation of EMSM method to it is briefly presented. Then based on the splitting strategy provided through ALE, the two-way coupling modeling and numerical strategies are discussed. Afterwards, some detailed comparisons are made between the proposed two-way coupled EMSM approach and a classical two-way coupled Lagrangian technique used in IFP-C3D software for injection simulations in engines. Finally, the approach is tested under realistic injection conditions: high velocities, real injectors with 3D geometry and small injection diameters.

Moreover, we remind that the present Eulerian model can only be used in the framework of sprays, that is not directly at the mouth of the injection process where primary atomization has to be described. The high order moment method can only be used once the spray is formed and this why, in the injection test-cases proposed hereafter, the injection velocity is representative of the velocity of the spray encountered at some distance from the nozzle where the liquid fuel has already been atomized and can be adequately considered as a spray.

\subsection{Adaptation to the Abritrary Lagrangian Eulerian (ALE) formalism}

\subsubsection{Overview of peculiarities introduced by the ALE formalism}

According to ALE, the complete resolution of conservation equation system is solved using a splitting strategy, where each stage corresponds to a class of physical phenomena. Such as in the standard Strang splitting method, the first stage is refereed as phase A since it is concerned with drag, heat and evaporation source terms in EMSM model. In case of a two-way coupled EMSM method, phase A contains also source terms in Navier Stokes equation system. The associated two-way coupling modeling and numerical strategy for robust and accurate simulations are presented in section 4.2. Unlike Strang method, in ALE, a new referential frame is introduced for the treatment of remaining terms in conservation equations, leading to (phase $B$ ) and (phase $C$ ) involving respectively acoustic and convective terms. Since we are in a pressure-less gas formalism in the context of EMSM method, the treatment of phase $B$ is straightforward, whereas the robust numerical method, preserving the moment space developed in [60] is used for the phase $C$ treatment [58]. For a better understanding of the EMSM under ALE formalism, a schematic of the implementation of the EMSM model in the IFP-C3D code structure is presented in Fig. (8). In the next part, a preliminary test-case verifying the adaptation of EMSM method to ALE formalism is presented.

\subsubsection{Elementary tests}

The objective of this test case is to ensure that the implemented model is stable with mesh movement through one-way coupling with gas phase (no effect of the spray on the gas motion). The evolution of homogeneous fields of liquid and gas are considered in a closed high-pressure cell. The bottom boundary of this cell corresponds to a moving piston at the bottom dead center. The gas is taken as air, and the particles are initially stationary. No ignition occurs, and no thermic effect is considered. Also, no special treatment of the boundary is considered. The computation ends after a revolution of the crank, with the crank angle degree (cad) ranging in $[-180,180]$. The high-pressure cell and the movement of the piston are described in Fig. (9).

The boundary conditions are $\left(\boldsymbol{u}_{\boldsymbol{p}}-\boldsymbol{u}_{\text {piston }}\right) \cdot \boldsymbol{n}=0$ on the surface of the piston, and $\boldsymbol{u}_{\boldsymbol{p}} \cdot \boldsymbol{n}=0$ at the upper edge of the domain. The size distribution is constant. During the compression and expansion of the highpressure cell, the computational cell volumes change is homothetic. The results are displayed for the number density $m_{0}$ and the surface density $m_{1}$ with a $1200(30 \times 40)$ cell grid. Computational results are presented in 


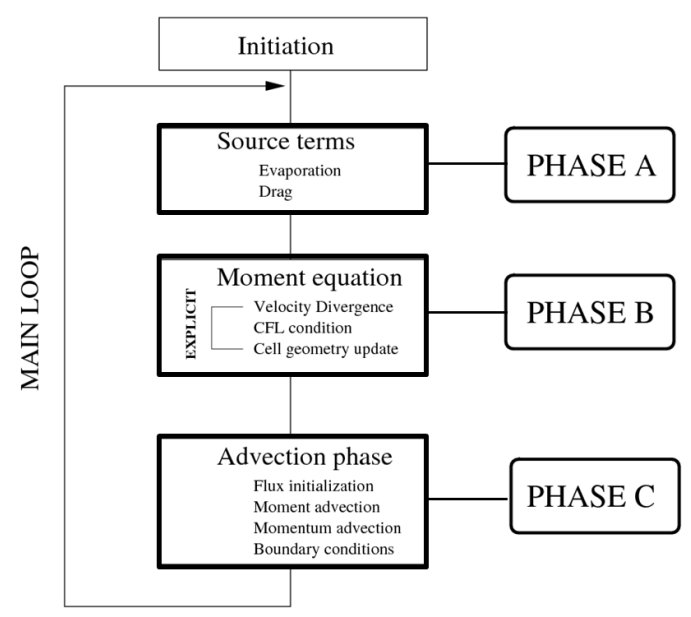

Figure 8. Scheme of the implementation of the EMSM in the operator-split structure of the IFP-C3D code
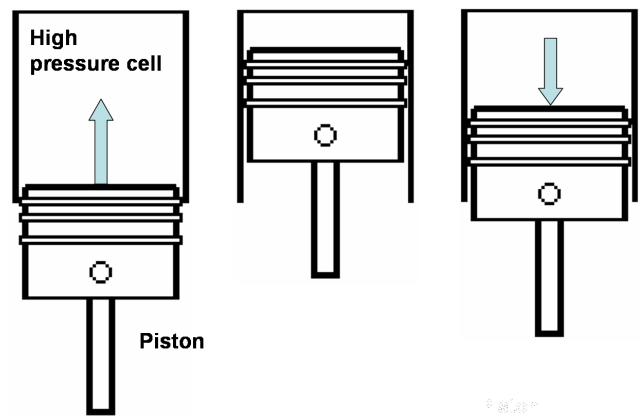

Figure 9. Considered piston movement during the computation. The computation starts at $c a d=-180$ and ends at $c a d=180$.

the case of a spray. Figure (10) displays the results for the moments in the case of a spray at different instants $c a d=-100,-30,50,180$. In the various subfigures, the distance where the value of the moments is null is the distance traveled by the piston. This is a typical case where the operator splitting done in the algorithm of IFP-C3D is legitimate since the speed of sound is at least one order of magnitude higher than the convective speed of the fluid. In fact, the speed of sound exceeds $300 \mathrm{~ms}^{-1}$. At the same time, with a rating of $1200 \mathrm{trmn}^{-1}$, and stroke of about $10 \mathrm{~cm}$, the piston average velocity and that of the dragged fluid is much smaller than the speed of sound in the gas.

The two first instants ( $c a d=-100$ and $c a d=-30$ ) correspond to the compression of the high-pressure cell. The two following ones $(c a d=50$ and $c a d=180)$ correspond to the expansion of the domain. Inertial particles in the infinite Stokes limit are considered, since no interaction with the gas phase is taken into account. They stick to the piston as it moves forward. This behavior is observed in Fig. (10)-top and is responsible for the singularity present at the piston surface. Meanwhile, the moment field downstream of the piston is unchanged. This is a direct consequence of the assumption made for pressure-less gas dynamics, wherein the speed of sound is null, and consequently no pressure waves modify the field. In Fig. (10)-bottom, the $\delta$-shock moves forward relative to the mesh. The enlargement of the $\delta$-shock has two explanations. The first one is the numerical diffusion. The second is that the computational cell volume expansion contributes to the $\delta$-shock enlargement. Meanwhile, upstream of the $\delta$-shock, in the wake of the piston, a vacuum zone is created, which is the expected behavior.

This test case assesses the robustness of the scheme implemented in IFP-C3D in the context of moving grids. This is a substantial achievement since one recalls that this scheme has to preserve two critical stability conditions: the realizability condition on the moments, and the discrete maximum principle on the spray velocity. This example amply illustrates that even within the algorithm of an industrial code, sensitive stability conditions can be respected. 

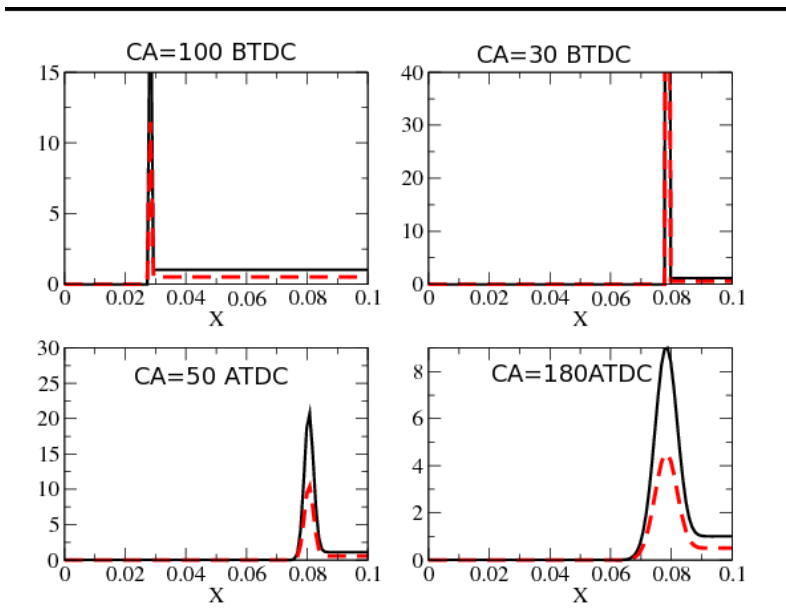

Figure 10. Results in the case of a polydisperse spray for $m_{0}$ (solid black line) and $m_{1}$ (dashed red line). Distance $X$ corresponds to the one-dimensional coordinate along which the piston moves : from left to right for subfigures on the top row (CA 100 BTDC and CA 30 BTDC) and from the right to the left for subfigures on the bottom row (CA 50 ATDC and CA 180 ATDC).

\subsection{Accounting for two-way coupling in stiff conditions}

So far, the high order moment method EMSM has been argued to be the best compromise in polydisperse spray modeling for our problem. Yet the underlying two-phase flow physics encountered in Internal Combustion Engines are driven through two-way mass, momentum and energy interactions between phases. It is therefore necessary to describe the presence of spray, while determining the gas-phase governing equations. Let us recall that the volume fraction $\alpha \ll 10^{-2}$ associated to the spray is small enough to describe the effect of the spray-phase on the gas-phase through source terms only. The gas-phase classically given by the compressible Navier Stokes equations is thus written down as

$$
\begin{aligned}
\partial_{t} \rho_{g}+\nabla_{\mathbf{x}} \cdot\left(\rho_{g} \mathbf{u}_{\mathbf{g}}\right) & =S^{\text {mass }} \\
\partial_{t}\left(\rho_{g} Y_{k}\right)+\nabla_{\mathbf{x}} \cdot\left(\rho_{g} Y_{k} \mathbf{u}_{\mathbf{g}}\right) & =\nabla_{\mathbf{x}} \cdot\left(\rho_{g} D_{k} \nabla_{\mathbf{x}} Y_{k}\right)+\dot{w}_{k}+S_{k}^{\text {species }} \\
\partial_{t}\left(\rho_{g} \mathbf{u}_{\mathbf{g}}\right)+\nabla_{\mathbf{x}} \cdot\left(\rho_{g} \mathbf{u}_{\mathbf{g}} \otimes \mathbf{u}_{\mathbf{g}}\right) & =-\nabla_{\mathbf{x}}\left(P_{g}\right)+\nabla_{\mathbf{x}} \cdot(\tau)+S^{m o m} \\
\partial_{t}\left(\rho_{g} E_{g}\right)+\nabla_{\mathbf{x}} \cdot\left(\rho_{g} E_{g} \mathbf{u}_{\mathbf{g}}\right) & =-\nabla_{\mathbf{x}} \cdot(\mathbf{q})-\nabla_{\mathbf{x}} \cdot\left(P_{g} \mathbf{I} \mathbf{u}_{\mathbf{g}}\right)+\nabla_{\mathbf{x}}\left(\tau \mathbf{u}_{\mathbf{g}}\right)+S^{\text {enth }},
\end{aligned}
$$

where source terms $S^{\text {mass }}$ and $S_{k}^{\text {species }}$ are the fuel vapor mass addition to the gas-phase due to the evaporation, $S^{m o m}$ is the momentum exchange terms between phases due to spray evaporation and drag, $S^{E_{\text {enth }}}$ is the total energy production in the gas due to the spray evaporation. The mass density $\rho_{g}$, the velocity $\mathbf{u}_{\mathbf{g}}$, the total energy $E_{g}$ and the pressure $P_{g}$ are quantities related to the gas-phase flow. The index $k$ denoting species involved in the gas, $Y_{k}$ and $D_{k}$ represent respectively the species mass fraction and molecular diffusion coefficient.

The expressions relative to the above mentioned source terms are naturally arisen through the integration of 
the kinetic model described by Eq. (10) and are thus given as

$$
\begin{aligned}
S^{\text {mass }}= & \iiint \frac{\rho_{d}}{4 \sqrt{\pi}} S^{1 / 2} f R_{S} d S d \boldsymbol{u} d T, \\
S_{k}^{\text {species }}= & \iiint \delta_{F u, k} \frac{\rho_{d}}{4 \sqrt{\pi}} S^{1 / 2} R_{S} f d S d \boldsymbol{u} d T, \\
S^{\text {mom }}= & -\iiint \frac{\rho_{d} S^{3 / 2}}{6 \sqrt{\pi}} \boldsymbol{D}_{r, \boldsymbol{p}} f d S d \boldsymbol{u} d T \\
& +\iiint \frac{\rho_{d}}{4 \sqrt{\pi}} u S^{1 / 2} f R_{S} d S d u d T, \\
S^{\text {enth }}= & \iiint \frac{\rho_{d}}{4 \sqrt{\pi}} S^{1 / 2} C_{p} R_{S} f d S d \boldsymbol{u} d T .
\end{aligned}
$$

Here $C_{p}$ is the specific heat capacity at constant pressure. In this example, $\delta_{F u, k}=1$ with $F u=k$ since droplets are exclusively composed of fuel molecules. We recall that, based on the monokinetic assumption previously introduced for the local spray velocity distribution, these source terms have been reconstructed through the maximum entropy reconstruction of the size distribution function $[35,58,36]$.

\subsubsection{Source term reconstruction and two-way coupled model}

Given the expressions (45), one can see that fractional moments are needed to close source terms involved in the gas phase. These are written as

$$
m_{k+1 / 2}=\int_{0}^{S_{\max }} \frac{S^{k+1 / 2}}{6 \sqrt{\pi}} \tilde{n}(t, S) d S, \quad k \in\{0,1\} .
$$

Even though the classical EMSM method requires only the transport of integer moments, fractional moments of the distribution can be reconstructed from the ME method. The accuracy of the $m_{3 / 2}$ moment reconstruction, accounting for mass concentration, is demonstrated in [82, 83]. This reconstruction technique is therefore chosen for our problem. Let us now give the new expression for the ODE system involving both the gas and the spray in phase A of ALE, under the $d^{2}$ type of evaporation law:

$$
\begin{aligned}
d_{t}\left(\rho_{g}\right) & =\frac{3}{2} m_{1 / 2} R_{s} \\
d_{t}\left(\rho_{g} u_{g}\right) & =-m_{1 / 2} \frac{u_{g}-u_{d}}{\tau_{p}^{\circ}} S_{\max }+\frac{3}{2} m_{1 / 2} R_{s} u_{d}
\end{aligned}
$$

where $\tau_{p}^{\circ}=\rho_{d} S_{\max } /\left(18 \pi \mu_{g}\right)$ is the characteristic dynamic time scale associated to the maximum sized droplet $S_{\max }$ in the spray. The evolution of the gas-phase internal energy $e_{g}$ due to evaporation is

$$
d_{t}\left(\rho_{g} e_{g}\right)=\frac{3}{2} m_{1 / 2} R_{s} e_{d}
$$

where $e_{d}$ the spray-phase internal energy. Let us recall that the evolution of the spray velocity is accounted by the equation:

$$
\partial_{t}\left(m_{1} u_{d}\right)=m_{0} \frac{u_{g}-u_{d}}{\tau_{p}^{\circ}} S_{\max }-R_{S} m_{0} u_{d}
$$

However, one can also write the previous equation in the following way to guarantee automatically the momentum conservation in phase A:

$$
\partial_{t}\left(m_{3 / 2} u_{d}\right)=m_{1 / 2} \frac{u_{g}-u_{d}}{\tau_{p}^{\circ}} S_{\max }-\frac{3}{2} R_{S} m_{1 / 2} u_{d}
$$




\subsubsection{Description of the proposed strategy}

In our problem the two-way interactions occur under stiff conditions in an internal combustion engine which involve rapid variations related to mass, momentum and energy exchanges. Moreover, associated physical time scales, depending on droplet size variable, are becoming smaller and smaller because of the evaporation phenomenon. Under such constraints, the numerical stability is required to be unconditionally guaranteed while the moment space is conserved. An accurate and stable resolution of System (34) based on a kinetic scheme, has been developed in [83], preserving the moment space. The latter has been proven to be of at least first order of accuracy but with small numerical errors making it very accurate [36]. Conserving the spray evaporation scheme as developed in [83], a stable and accurate two-way coupling numerical strategy for the coupled resolution of System (34a)-(34d), Eq.(47), Eq.(48), Eq.(51) and Eq.(49) has been recently developed in [35, 58] and proved to preserve moment space. In order to guarantee the numerical stability under small dynamic time scales for droplets, a third order A-stability and L-stability singularly diagonally implicit Runge Kutta method (SDIRK)[49] has been used for the coupled resolution of Eq.(48) and Eq.(51). The time integration of SDIRK within a time step is illustrated in Figure 11. Let us recall that although the scheme is originally developed for constant d-square evaporation law, its extension to more realistic laws such given in [1] has been also achieved through preliminary works [36, 33].

In the next section, both qualitative and quantitative evaluation of the two-way coupling strategy will be investigated.

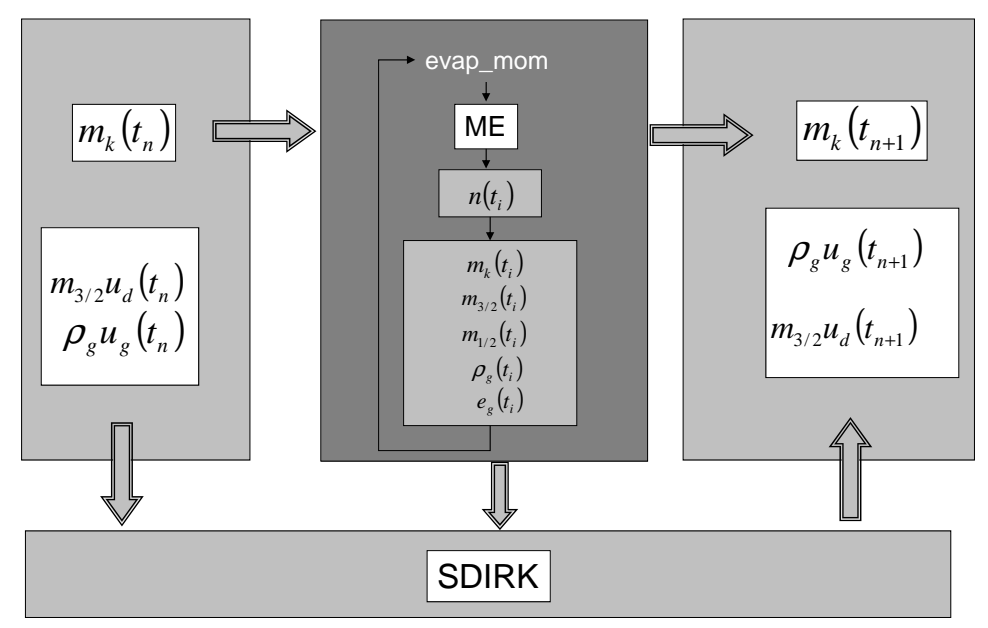

Figure 11. Illustration of the mass and momentum two-way coupled exchanges resolution between the evaporating spray and the gas in phase A of the ALE solver (ME stands for Entropy Maximisation, $t_{i}$ are the intermediate timings for the resolution of the Runge Kutta steps between $t_{n}$ and $t_{n+1}$ ).

\subsubsection{Evaluation strategy of the method}

As mentioned before, the two-way coupling method is intended to handle stiff injection conditions, guaranteeing robustness and accuracy of numerical solutions in realistic applications. Therefore the next section 4.3 is dedicated to verify these claimed features with the help of test cases through IFP-C3D code.

- In section 3, we exclusively handle DNS Eulerian models for the description of a polydisperse spray and its two-way coupling with a turbulent flow field. However, since we want to evaluate the behavior of the model in the context of internal combustion engines, for which the present interest and implementation relies on the code IFP-C3D, we have implemented the models and numerical schemes in the IFP-C3D code. In the case of spray injection, a turbulent model for the gaseous flow field is needed and we rely on a RANS approach purely for the unsteady gaseous flow field, since no model is presently available which is consistent with polydisperse spray dynamics and two-way coupling at a RANS level, except a very recent contribution [34]. 
Switching to a comprehensive and consistent two-way coupled RANS model would require a much deeper investigation in terms of mathematical structure of the resulting systems of equations and the development of new numerical strategies. The two-way coupling is thus conducted in the chosen configurations on the non-fluctuating quantities only, for both Lagrangian and Eulerian models.

- First, the accuracy and the stability of the solutions under different Stokes regimes, typical to ICE conditions are investigated through comparisons to widely used existing two-way coupled Lagrangian Stochastic Parcel method in IFP-C3D software. In that case, instantaneous mass, momentum and energy exchanges between phases are resolved through the two-way coupling equation system presented in part 4.2.

- Second, the robustness of the method under realistic internal combustion engine conditions, taking into account typical injection velocity and injection conditions, is investigated. This implies a fully turbulent two-phase flow. To treat turbulence physics in the gas-phase, a standard $k$ - $\varepsilon$ (RANS) model is available in IFP-C3D. Although our method derived in part 4.2 does not allow us to describe the two-way coupling in terms of fluctuating quantities but only mean quantities, it is still important to verify the method within the presence of a standard $k-\varepsilon$ gas model. Let us recall that only the robustness can be investigated since a turbulence model such as the one derived in [34] is required for a validation against experimental results.

\subsection{Injection test cases}

The first test case is chosen such that one has the corresponding experimental data for a thin, polydisperse and collisionless spray under the turbulent dispersion of droplets [37]. However, we will limit our study to a laminar flow regime. The test case consists of a $2 \mathrm{D}$ configuration presented in Fig. (13). This is proportional to a 2D mesh in the $y$ and $x$ directions. This $2 \mathrm{D}$ plane is composed of square cells with a length of $0.25 \mathrm{~mm}$ (320 in the $x$-direction, and 800 in the $y$-direction). However, two cells with a length of $0.5 \mathrm{~mm}$ along the $z$-axis had to be kept since IFP-C3D software is a 3D code. The $32 \times 16$ cell additional part at the top of the mesh is dedicated to the injection type inlet condition. Periodic boundary conditions are set on faces orthogonal to the $z$-axis, and free exit boundary conditions are set for the part localized at $y=0, x=40 \mathrm{~mm}$ and $x=-40 \mathrm{~mm}$. The cases presented in this section involve injection of a fuel spray and gas with velocity $u_{i n j}=18 \mathrm{~m} / \mathrm{s}$. The gas phase is taken as air, with initial pressure $P_{g}=10^{5} \mathrm{~Pa}$, temperature $T_{g}=293 \mathrm{~K}$, and viscosity $\mu_{g}=1.99 \times 10^{-5} \mathrm{~kg} / \mathrm{m} / \mathrm{s}$, whereas the fuel is taken as the $\mathrm{C}_{14} \mathrm{H}_{30}$ species, with a density $\rho=763 \mathrm{~kg} / \mathrm{m}^{3}$. Some other initial thermodynamic quantities can be found in Table 1 .

\begin{tabular}{l|ll}
$C_{p, g}$ & 1014.04 & $\mathrm{Jkg}^{-1} \mathrm{~K}^{-1}$ \\
\hline$C_{v, g}$ & 724.66 & $\mathrm{Jkg}^{-1} \mathrm{~K}^{-1}$ \\
\hline$\gamma_{g}$ & 1.399 & - \\
\hline$e_{g}$ & 21318.25 & $\mathrm{Jkg}^{-1}$ \\
\hline$R_{g}$ & 8.314 & - \\
\hline$C_{v}$ & 1435.91 & $\mathrm{Jkg}^{-1} \mathrm{~K}^{-1}$ \\
\hline$e$ & -59784.88 & $\mathrm{Jkg}^{-1}$
\end{tabular}

Table 1. Values of heat capacity in constant pressure of the gas $C_{p, g}$, in constant volume of the gas $C_{v, g}$ and the fuel spray $C_{v}$. Specific internal energies of the gas $e_{g}$ and the spray $e$, the perfect gas constant $R_{g}$ and the ratio $\gamma_{g}=C_{p, g} / C_{v, g}$.

We introduce a characteristic length $L_{0}=8 \times 10^{-3} \mathrm{~mm}$, which is the injection diameter and the corresponding time $\tau_{g, 0}=L_{0} / u_{i n j}$ for the gas flow. In our case, the mean droplet diameter being significant for the study of the disperse phase, the characteristic dynamic time scale for the droplet population is given as a function of the SMR 
number:

$$
\tau_{0}=\frac{2 \rho r_{\mathrm{SMR}}^{2}}{9 \mu_{g, 0}}
$$

We also define some dimensionless numbers such as the fractional volume occupied by the dispersed phase $\Phi_{\mathrm{v}}$ and the Stokes number St expressed as the ratio of the droplet dynamic time response $\tau_{0}$ over the gas characteristic time $\tau_{g, 0}$.

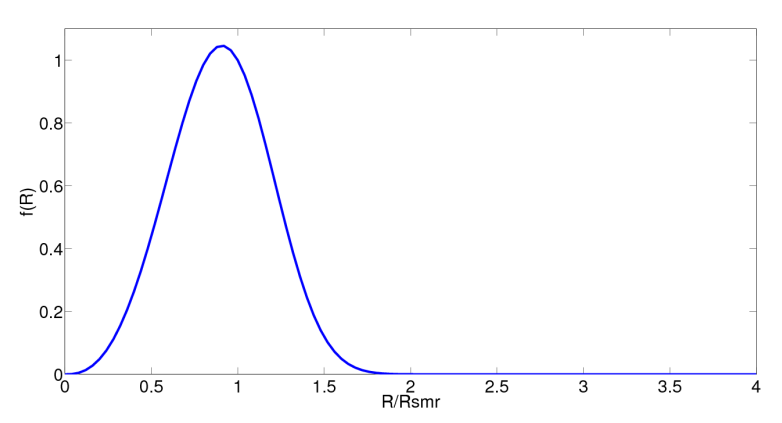

Figure 12. Illustration of Rosin-Rammler distribution used in all the multidimensional computations.

The computations are run with a polydisperse droplet population governed by the Rosin-Rammler distribution [102]:

$$
f(r)=q \frac{r^{q-1}}{r_{\mathrm{SMR}}^{q}} \exp \left(-\frac{r^{q}}{r_{\mathrm{SMR}}^{q}}\right)
$$

where $r$ is the particle radius, $r_{\mathrm{SMR}}$ is the Sauter mean radius, and $q$ is a coefficient determining the sharpness of the distribution. The distribution considered here has $q=3.5$, see Fig. (12).

\subsubsection{Eulerian spray initialization}

One needs to ensure that identical boundary conditions are applied for both Eulerian and Lagrangian simulations. Given the value of injected liquid volume fraction $\Phi_{\vee}$ and the distribution function shown in Fig. 12, one can compute moments of the distribution at injection for the Eulerian simulations. However, the injection of Lagrangian particles is controlled by the total liquid mass $m_{l}$, the injection time length $t_{i n j}$ and the injector radius $r_{i n j}$. To have the same injection conditions in Lagrangian cases, we first compute the mass flow rate for a given inlet velocity and inlet volume fraction by the formula

$$
\dot{m}_{l}=\Phi_{\mathrm{v}} \rho u_{i n j} S
$$

with $S$ the injection section for the two-phase flow and $\rho S$ corresponds to the injection section for the Lagrangian particles. From the latter, $r_{i n j}$ can be easily calculated. Moreover, from the knowledge of $t_{i n j}, m_{l}$ is deduced from

$$
m_{l}=\dot{m}_{l} t_{i n j}
$$

Lagrangian particles are injected in the middle of each cell face belonging to the inlet condition, as sketched out in Fig. 13. 64 cells at the top of the mesh leads to a total of 64 injectors. Since the mesh is a 2-D plane, the radius and the injection section related to each injector remain the same.

\subsubsection{Comparisons between Eulerian and Lagrangian simulations}

In the context of injection simulations, the two-way coupling effect on flow dynamics can not be neglected when the mass ratio of the dispersed phase over the gas phase becomes significant. Moreover, inertial droplets inside a distribution have a slow response to changes in the gas whereas light particles barely follow the carrier flow. The spray dispersion is thus influenced by the size of droplets. In internal combustion engines, a broad range of 


\begin{tabular}{c|cc}
$r_{\mathrm{SMR}}$ & $5 \mu \mathrm{m}$ & $20 \mu \mathrm{m}$ \\
\hline $\mathrm{St}$ & 0.48 & 7.67 \\
\hline$\Phi_{\mathrm{v}}$ & $2.51 \cdot 10^{-4}$ & $2.51 \cdot 10^{-4}$ \\
\hline$\Phi_{\mathrm{m}}$ & 0.19 & 0.19 \\
\hline$\tau_{v}$ & $1.4 \cdot 10^{-2} \mathrm{~s}$ & $1.4 \cdot 10^{-2} \mathrm{~S}$ \\
\hline$\tau_{v} / \tau_{0}$ & 65.7 & 4.1 \\
\hline $\operatorname{Re}$ & 7200 & 7200
\end{tabular}

Table 2. Dimensionless characteristic values at injection: Stokes numbers St, spray volume fraction $\Phi_{\mathrm{v}}$ and mass loading $\Phi_{\mathrm{m}}$ are computed through the Sauter mean radius $r_{\mathrm{SMR}}$.

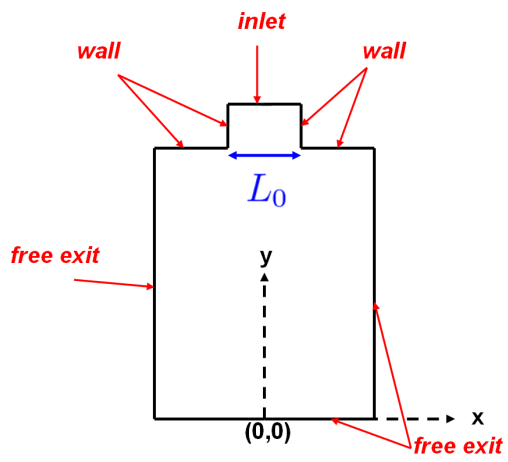

Figure 13. Illustration of the 2D plane geometry - The injection domain is $20 \mathrm{~cm}$ depth and $8 \mathrm{~cm}$ width.

fuel droplet sizes needs to be considered. We run injection simulations under two types of droplet distributions characterized by different Sauter mean radius $r_{\mathrm{SMR}}$ but the same mass loading $\Phi_{\mathrm{m}}$ or volume fraction $\Phi_{\mathrm{v}}$, as observed in Table 2. These initial characteristic numbers are chosen such that droplet-droplet interactions can be assumed to be neglected. Note that we adopt a simple $d^{2}$ evaporation law of droplets under a constant evaporation time $\tau_{v}$ for both low and high inertia droplets. The small ratio of the evaporation time over the characteristic droplet time $\tau_{v} / \tau_{0}$ shows that the spray with high inertia droplets undergoes a faster evaporation.

For each distribution, we run both Lagrangian and full Eulerian resolutions of the disperse flow in exactly the same conditions. The initial spray mass flow rate is taken as $2.758 \times 10^{-5} \mathrm{~kg} / \mathrm{s}$. A total number of 6.4 million numerical particles, insuring the convergence of the Lagrangian solution, injected to the domain. In the following, results are displayed for a simulation time of $1.4 \times 10^{-2} \mathrm{~s}$. The latter also corresponds to the characteristic evaporation time $\tau_{v}$, as shown in Table 2. The results presented in the following are for evaporating cases. More details about the tests in non-evaporating conditions can be found in [36].

Injection of low inertia droplets Injection results in case of light droplet population with Sauter mean radius of $r_{\mathrm{SMR}}=5 \mu \mathrm{m}$ are illustrated in Figs. 14 and 15. The fields of spray volume fraction and spray velocity for both Lagrangian and Eulerian sprays are quite similar. The global droplets dynamics are well reproduced. Let us note that the front of the Lagrangian spray is sharper than the Eulerian one.

The vapor fuel along with the temperature fields are also very well represented, as seen in Fig. 15. This shows that the mass transfer and temperature modification in the gas due to the evaporation between phases are well captured through the two-way coupling algorithm. Moreover, the gas velocity field driven by the Eulerian spray is 
qualitatively similar to the one driven by the Lagrangian spray, see Fig. 14. There is a good quantitative comparison between both approaches.
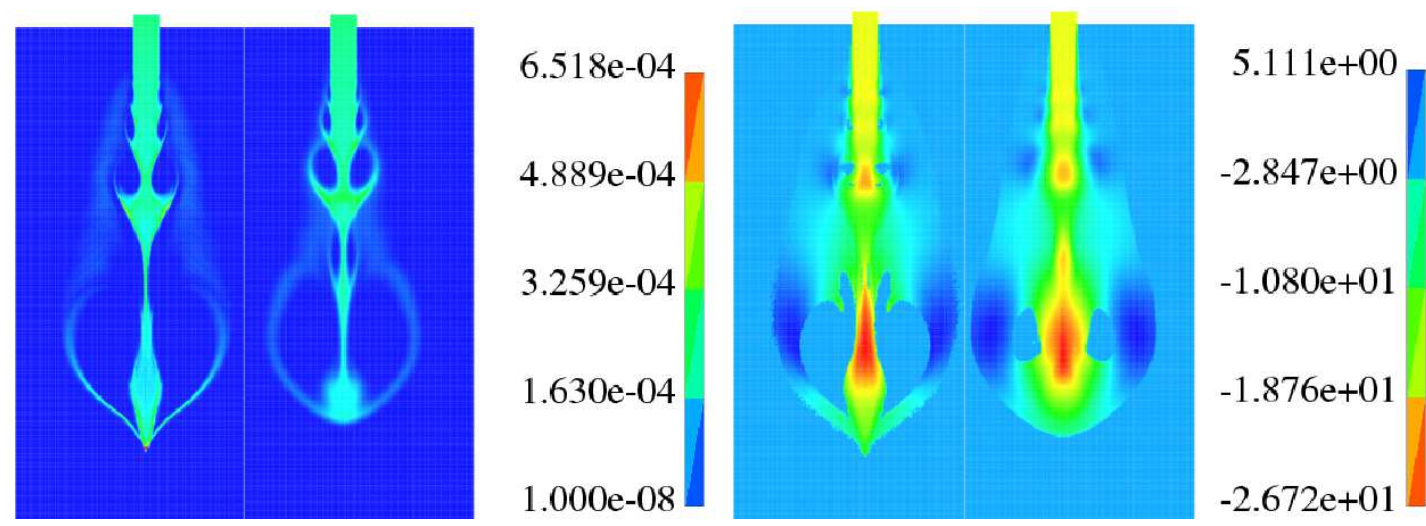

Figure 14. Results for an evaporating spray with a droplet population of $r_{\mathrm{SMR}}=5 \mu \mathrm{m}$ at time $1.4 \times 10^{-2}$ s. Left: Spray volume fraction.Right: spray velocity along the $y$-axis. In each panel, Lagrangian spray is displayed on the left side and Eulerian on the right side.
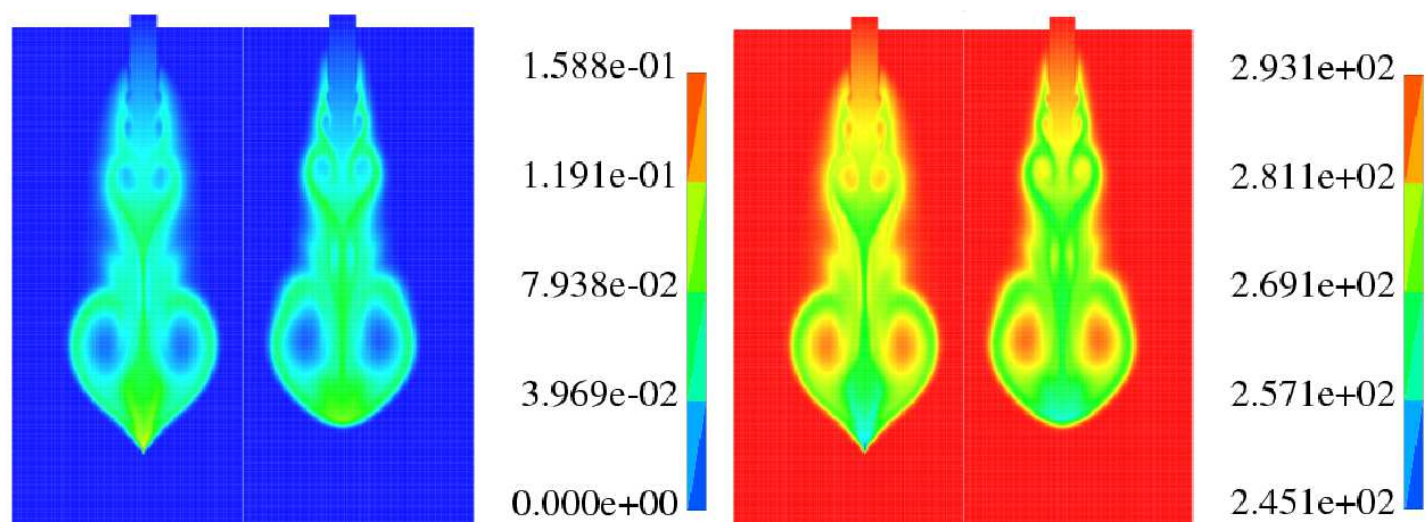

Figure 15. Results for a droplet population of $r_{\mathrm{SMR}}=5 \mu \mathrm{m}$ under a constant evaporation velocity at time $1.4 \times$ $10^{-2}$ s. Left: Evaporated fuel mass fraction. Right: Gas temperature. In each panel, Lagrangian spray is displayed on the left side and Eulerian on the right side.

Injection of high inertia droplets In case of a droplet population with $r_{\mathrm{SMR}}=20 \mu \mathrm{m}$, results from fields of spray volume fraction, spray velocity, gas velocity are illustrated in Fig. 16, whereas Fig. 17 shows the fields of evaporated fuel mass fraction and gas temperature. Through spray velocity and spray volume fraction fields, significant differences between Eulerian and Lagrangian sprays can be observed. There is a high concentration of Eulerian droplets at the front side of the spray. This situation can be understood through the fact that the pressureless gas formalism adopted for the spray modeling where the spray momentum equation is weakly hyperbolic without any diffusion terms. But this is not enough to explain the real situation since for low inertial droplets the pressure-less approach was working perfectly. The second issue is that in real situations, droplets with different sizes and different velocities can cross. Therefore, from the modeling point of view, the droplet trajectory crossing effect should be taken into account. Yet, the EMSM method has been shown to fail for that type of configuration. This failure stems from the assumption that all droplets located in the same cell have the same velocity. However, high inertia droplets cannot be considered to have the same velocity, since the drag force between phases is strongly conditioned by the droplet size. So one can conclude that the high droplet concentration observed for Eulerian spray leads to some unphysical solutions. Yet, the core of the Eulerian spray is quite similar to the Lagrangian one. Moreover, the high droplet accumulation is drastically reduced in case of evaporating droplets. This behavior is 

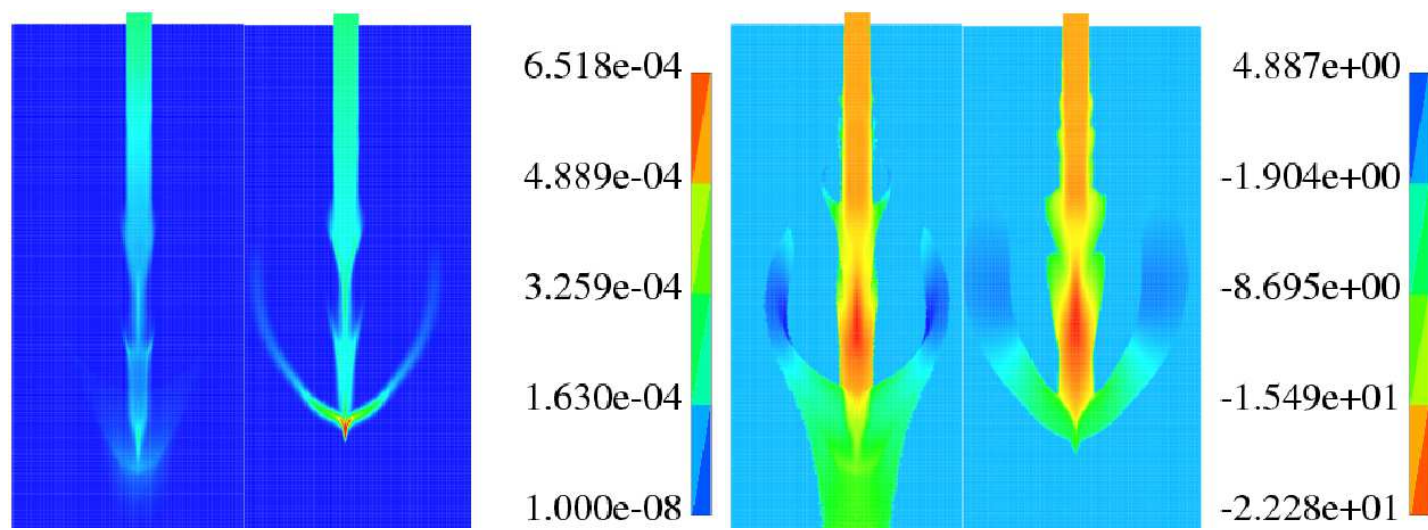

Figure 16. Results for an evaporating spray with a droplet population of $r_{\mathrm{SMR}}=20 \mu \mathrm{m}$ at time $1.4 \times 10^{-2}$ s. Left: Spray volume fraction. Right: spray velocity along the $y$-axis. In each panel, Lagrangian spray is displayed on the left side and Eulerian on the right side.
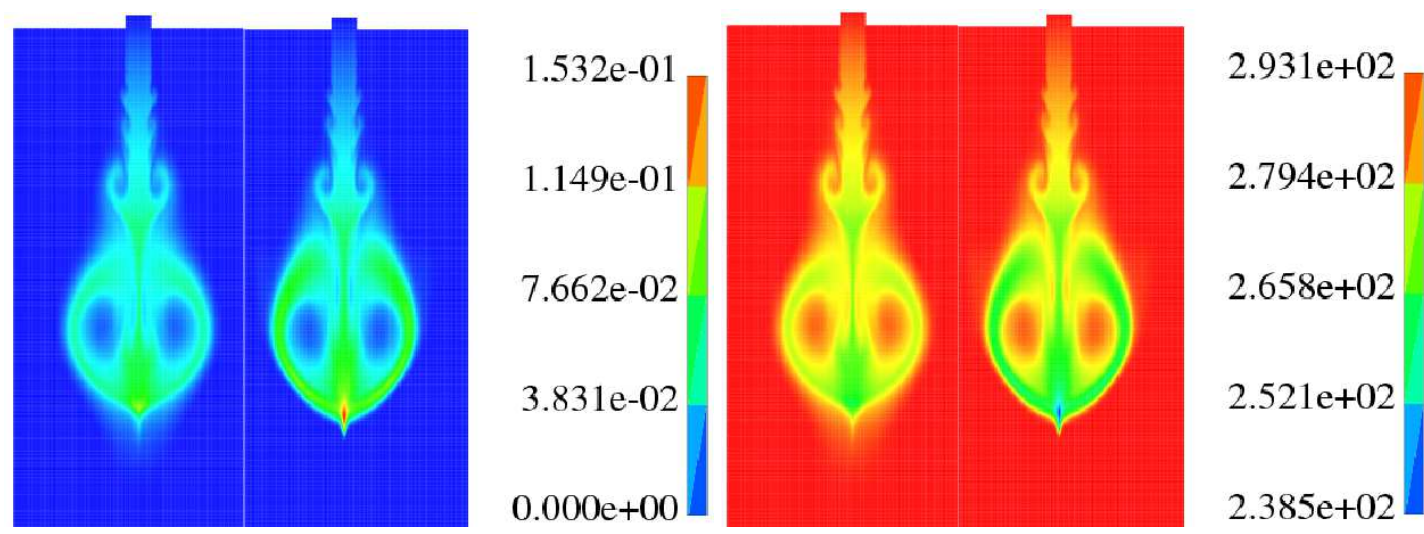

Figure 17. Results for a droplet population of $r_{\mathrm{SMR}}=20 \mu \mathrm{m}$ under a constant evaporation velocity at time $1.4 \times 10^{-2} \mathrm{~s}$. Left: Evaporated fuel mass fraction. Right: Gas temperature. In each panel, Lagrangian spray is displayed on the left side and Eulerian on the right side.

expected since evaporation leads to a decreasing droplet inertia for which the monokinetic assumption without the polydispsersity in velocity, works quite well. This in an encouraging situation for IC engine operating conditions, since the fuel droplet evaporation takes place under high temperatures and pressures. Fields of evaporated fuel mass fraction and the gas temperature between the Lagrangian and Eulerian sprays, as shown in Fig. 17, match quite well.

Moving mesh situations Representations of the spray radius showing the Lagrangian particules radius on the left side and the average smr $\left(r_{32}\right)$ deduced from the transported moments computed with EMSM on the right side show the fundamental differences between the two formalisms. Moreover Fig. 18 shows that the reduced information of the moments distribution succeeds in getting the correct qualitative and quantitative behavior of the spray dynamics even in the case of a moving mesh situation where the bottom part of the geometry features an engine piston characteristic kinematics at a velocity of $2000 \mathrm{rpm}$ with a $0.1 \mathrm{~m}$ stroke.

However some differences can be observed between Lagrangian and Eulerian simulations. They can be attributed to two origins, the first being the level of modeling assumptions, in terms of velocity conditioned on droplet size, which is different between the two simulations. Indeed, we can clearly see that, even if a good level of agreement is reached, the size-conditioned dynamics of the droplets can not be reproduced with the present original EMSM model. The CSVM model developed by A. Vié [122] would have the capability to handle it as explained in section 3.7.6. The second is related to the fact that IFP-C3D has some limitations in terms of Lagrangian capabilities (stochastic boundary condition injection of the polydisperse spray, two-way coupling treatment on un- 
structured meshes, ...) inherited from previous developments. In this context the Lagrangian simulations offer a reference solution in order to verify the proposed new Eulerian strategy, but a fully quantitative validation and evaluation of the convergence of the Eulerian method as compared to the Lagrangian one on the 2D injection configuration is beyond the scope of this work, and in particular our Lagrangian approach does not allow to conduct a convergence study.

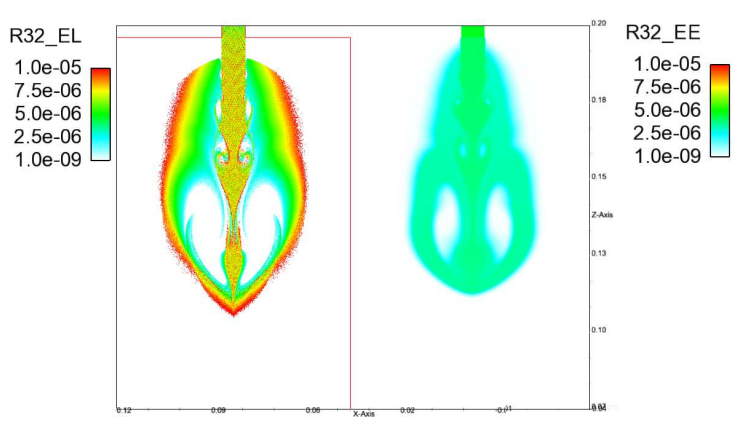

(a) 30 BTDC

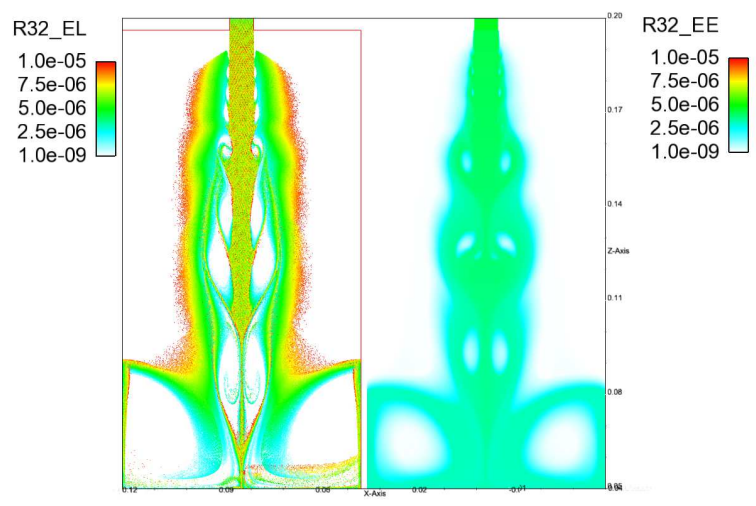

(b) 70ATDC

Figure 18. Comparisons between a Lagrangian simulation (left side) and the equivalent Sauter mean radius reconstructed from the transported moments (right) of an evaporating spray injected at a moderate velocity of 18 $\mathrm{ms}^{-1}$ in a moving mesh situation featuring an engine speed of $2000 \mathrm{rpm}$ and a stroke of $0.1 \mathrm{~m}$ (other dimensions are the same as those used in the previous simulations). Visualisations at crank angle 30 before top dead center $\mathrm{CA}=30$ degrees BTDC (a)- and at crank angle 70 after top dead center - CA = 70 degrees ATDC (b).

\subsubsection{High pressure injection conditions}

This test case is intended to assess the capacity of the EMSM model to be operated under realistic injection conditions for practical Diesel applications. As stated in Section 3, spray-gas turbulent interactions are not developed at this stage. The 3D injection computations will be conducted in a RANS framework for the gas by using a standard $k-\varepsilon$ approach for turbulent diffusion of gaseous related quantities. As stated in introduction, the turbulence generation in this high velocity jet configuration is only the result of the air motion due to the two-way coupled spray-gas macroscopic interactions. The test case features a 3D simulation of a $200 \mu \mathrm{m}$ diameter single hole injector inside a cylinder (60 mm diameter and $60 \mathrm{~mm}$ height), typical of a test cell geometry for actual Diesel injectors, which implies very different spatial scales. The mesh refinement is illustrated in Fig. 19. The smallest cells size inside the injector are around $\pm 5 \mu \mathrm{m}$.

The spray is injected at a velocity of $100 \mathrm{~ms}^{-1}$ in a quiescent ambient atmosphere $\left(P_{c h}=1 \mathrm{bar}, T_{c h}=293 \mathrm{~K}\right)$. This introduces very stiff conditions due the strong gradients related to the dynamics of injection and to the density ratios between the liquid and the surrounding gas. The fully coupled EMSM model described in the previous sections is tested under these conditions. The standard $k-\varepsilon$ model is activated in the gaseous phase. The volume fraction at the injection boundary is that of a disperse spray $\left(\Phi_{v} \simeq 10^{-2}\right)$ as the physics represented by the model does not include any dense effects at this stage. One can notice that, in spite of the very stiff conditions, the model remains stable. The smallest time steps are of the order of $10^{-8} \mathrm{~s}$ and the computation lasts 16 hours (on 128 processors) for a total physical time simulated of $4 \mathrm{~ms}$.

Results in Figs. 20 and 21 show how the spray develops from the onset of injection where a disperse phase assumption is made down to the fully developed spray region in the far field of the injector. Figure 20 shows the liquid volume fraction spatial distribution very close to the injector outlet. A typical cone shape distribution is observed due to the mixing with the surrounding gas. The qualitative aspect of the injected spray distribution and evaporated fuel distribution matches those issued from observations of classical high-pressure injected spray. Due to the two-way coupling method described above, the high velocity liquid jet generates turbulent energy in the gas 


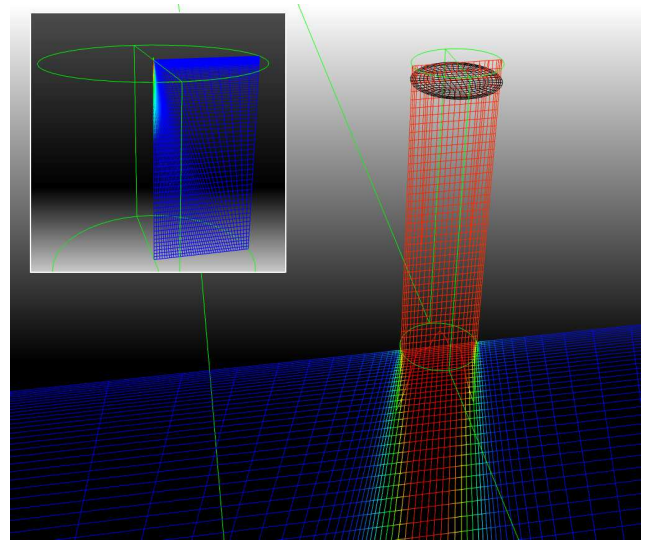

Figure 19. Injector hole geometry and chamber. Notice the refined boundary condition of the injection device revealing the multiscale nature of this case.

through macroscopic air entrainment at the tip of the injector (Fig. 22).

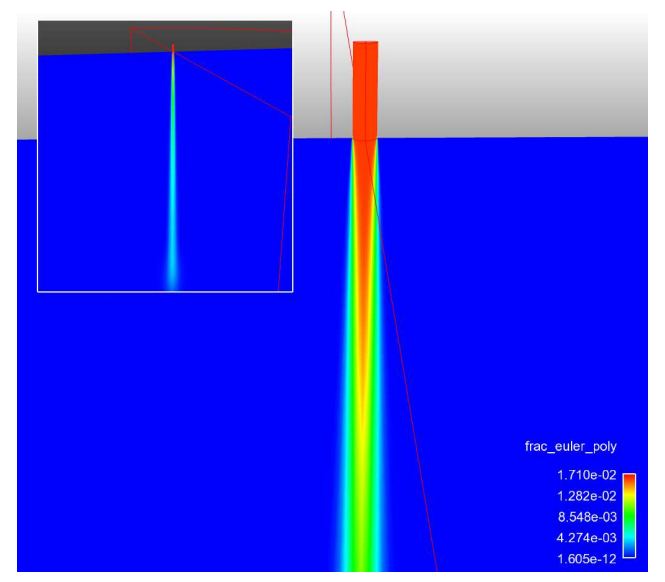

Figure 20. Eulerian volume fraction of liquid computed with the EMSM approach.

Figure 23 represents the evolution of the liquid and vapor concentrations at different axial positions. One can notice an opening of the spray jet along the injection direction. The fuel vapor distribution follows that of the liquid with higher concentrations on the jet axis. One can observe that vapor is entrained in the periphery of the jet which can be attributed to the turbulent transport of gaseous species. Figure 24 confirms that the highest vapor concentrations are located in the front of the jet whereas the liquid concentration continuously decreases along the jet axis under the evaporation process. A higher vapor concentration is observed in the near injector region and can be explained by the evaporation of the smallest droplets.

From these results, one can deduce penetration lengths for the liquid and for the gas that are the relevant quantities of practical interest for engine simulations. Figure 25 presents the liquid penetration based on the average liquid volume fraction of the polydisperse spray. It is calculated at an axial position where $10^{-3}$ of the maximum value of the volume fraction is reached. Different initial conditions on the spray volume fraction are tested with variation between a low volume fraction $\Phi_{\mathrm{v}}=10^{-4}$ to a higher volume fraction $\Phi_{\mathrm{v}}=10^{-2}$. For this last case two combinations of Sauter mean radius and droplet number are tested resulting in the same spray volume fraction: $\left(\mathrm{SMR}=20 \mu \mathrm{m}, \mathrm{N}=5.05810^{11}\right)$ and $\left(\mathrm{SMR}=5 \mu \mathrm{m}, \mathrm{N}=3.2310^{13}\right)$. One can notice that for these last two conditions the spray penetration is much more important as the overall inertia of the spray is higher. In this case high inertia case, the penetration is most important for the spray having the largest droplets. This is what would be expected on a qualitative level.

The two-way coupled numerical approach described above is currently being extended to handle complex evaporation and drag laws which are classically used in engine spray conditions. This will permit quantitative comparisons with real spray measurements and the next validation steps are the subject of these ongoing developments of realistic nonlinear laws for drag and evaporation to compute thermodynamic conditions relevant for 


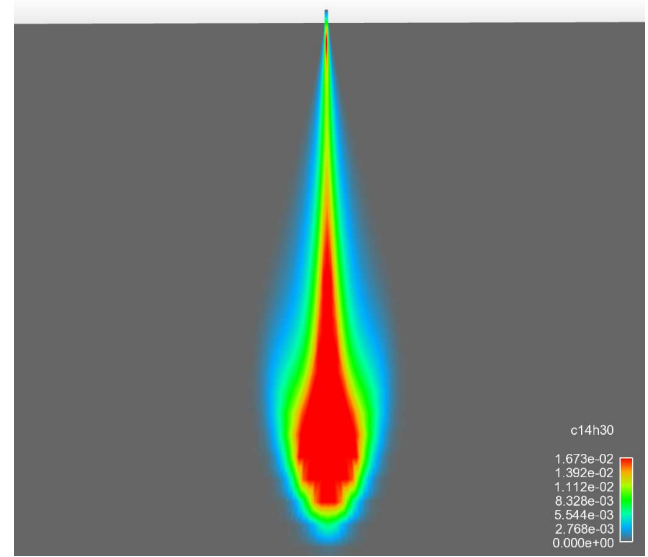

Figure 21. Gaseous fuel mass fraction evaporated with the EMSM approach.

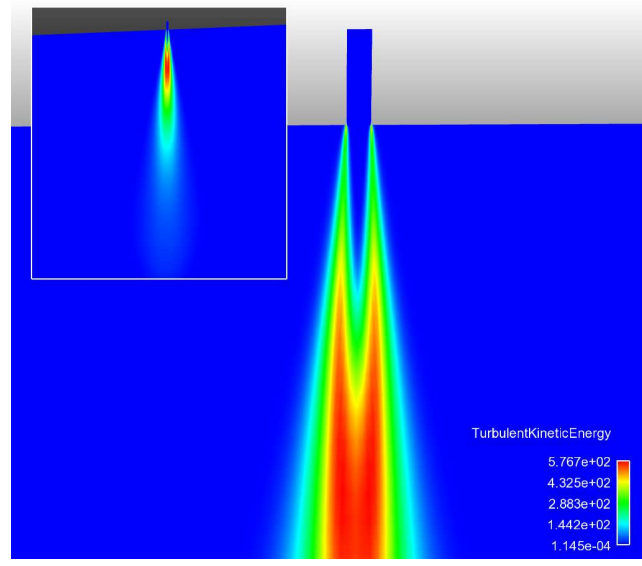

Figure 22. Gaseous turbulent kinetic energy related to high jet velocities.
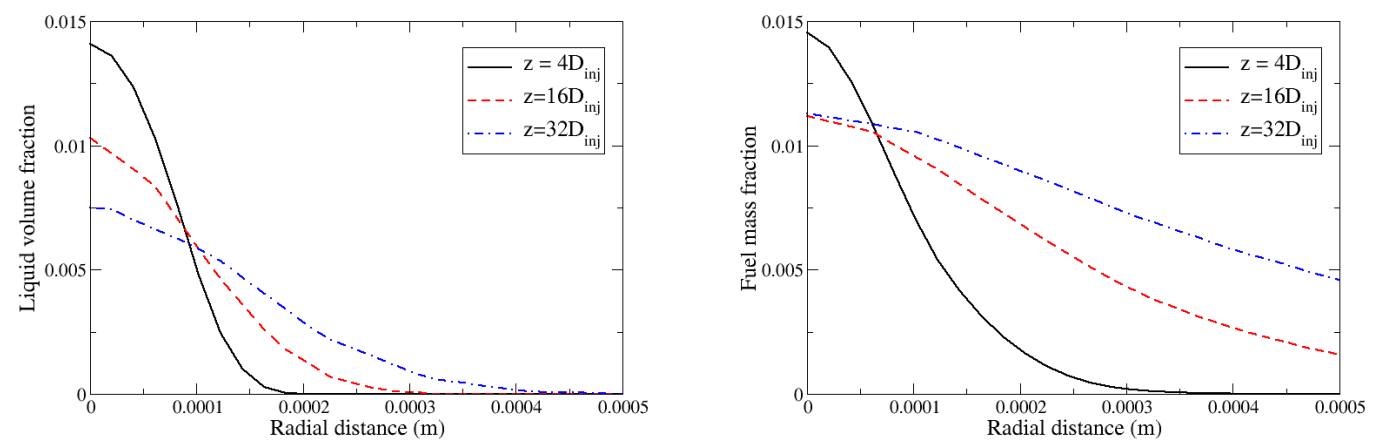

Figure 23. Radial profile of liquid volume fraction (left) and evaporated fuel mass fraction (right) at different axial positions: $z=4 D_{i n j}, z=16 D_{i n j}, z=32 D_{i n j}$. 

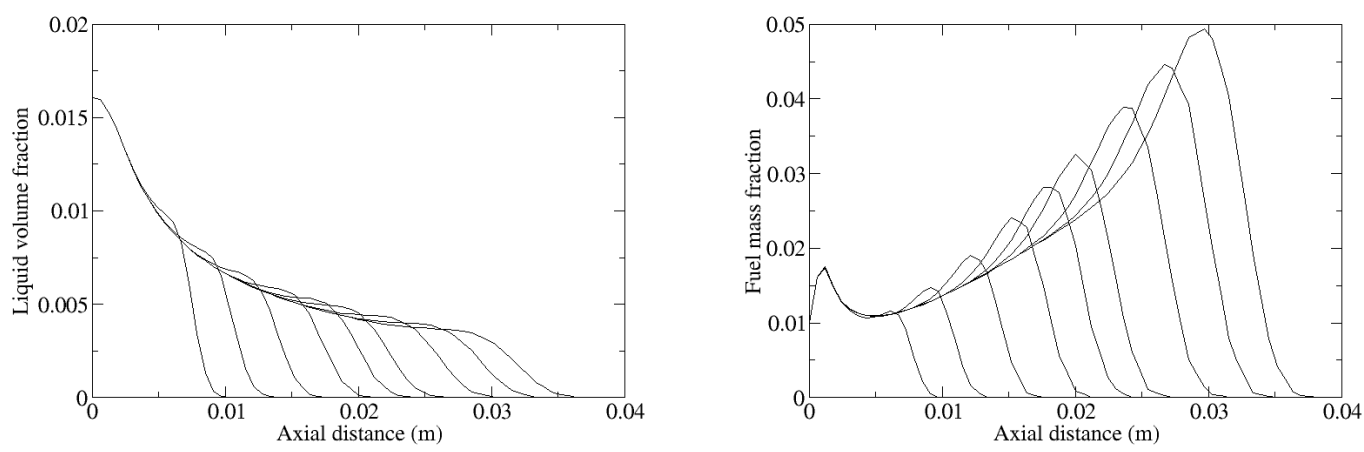

Figure 24. Axial profile of liquid volume fraction (left) and evaporated fuel mass fraction (right) at different consecutive instants between $t=0$ (start of injection) and $t=2.4 \mathrm{~ms}$.

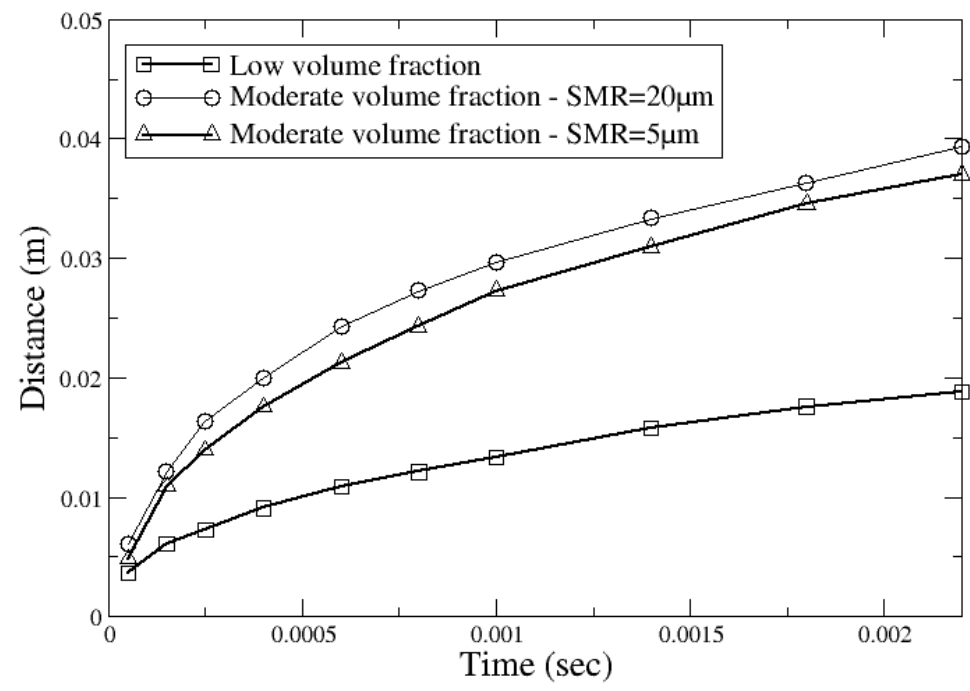

Figure 25. Liquid jet penetration with time for different initial conditions on charge: low charge $\left(\Phi_{\mathrm{v}}=10^{-4}\right)$ moderate charge $\left(\Phi_{\mathrm{v}}=10^{-2}\right)$ with two $r_{\mathrm{SMR}}$ values : $5 \mu \mathrm{m}$ and $20 \mu \mathrm{m}$.

engine applications.

\subsection{Concluding remarks on injection simulations}

In this section, the important issue of the ability of EMSM moment method to tackle the two-way polydisperse interactions of droplets with the surrounding gas phase has been revealed. The method has proven to be stable and accurate, preserving the moment space, within the multi-dimensional context and moving grid algorithms. Through its rigorous comparison with the widely used Lagrangian method and computations of realistic injection conditions, promising results towards IC engine applications were obtained. The good accuracy on fuel vapor field and gas phase properties shows that the Eulerian method is almost on equal footing with the Lagrangian method. The stability of the approach was assessed by full 3D computations with real injector meshing and stiff velocity and density gradients. This point is of crucial importance for the future development and for the practical use of Eulerian approaches. 


\section{Conclusions and future directions}

Throughout this paper, we have explained how to employ Eulerian methods and described the successive steps of development of an Eulerian moment approach for automotive injection purposes including their associated numerical method from fix to moving grids, and from one-way to two-way coupling. The main challenge was to get high order methods while ensuring strong stability conditions leading to robustness during the whole development process. A significant part of this work has consisted of implementing and validating the EMSM model in the IFPC3D code, an industrial software solving reactive compressible flows on unstructured grids including numerical algorithms and meshing facilities to deal with complex geometries and numerous specific models to deal with multiple interacting phenomena. By extensive numerical simulations of increasing complexity, it was shown that injection cases can be handled in an Eulerian framework.

Using specific examples, we have shown that high-order moment methods are able to describe polydispersity without discretizing the size phase space into sections, contrary to multi-fluid methods. The limitations of existing methods present in the literature [91, 41] were removed permitting their use in the applications we target. In this respect, the EMSM model can be considered a breakthrough both in terms of modeling, with a new and efficient way to describe polydispersity [83], and in terms of numerical methods, with a low level of artificial dissipation while maintaining the realizability condition and the maximum principle [60].

We give below an overview of our ongoing work to improve moment methods and their use for spray injection applications:

- Other important developments in relation to size-velocity coupling will be included to widen the range of applicability of moment methods for IC engine applications. Some issues have already been investigated on a fundamental level but extensions and applications to real injection conditions still need to be considered. For instance, resolving one additional moment transport equation, Vié et al. [122] have introduced the ability to describe a correlation between the size and velocity variables, leading to the coupled size and velocity moment (CSVM) model. Higher Stokes numbers can be reached with this approach. Other extensions of moment methods for particle crossing trajectories and their coupling with size polydispersity [118] could also be introduced based on theoretical derivations obtained in the $\mathrm{PhD}$ thesis of Damien Kah.

- Another step for the target applications is to extend the two-way coupled numerical approach to handle complex evaporation and drag laws, which are required to deal with IC engine spray situations. These aspects are currently under investigation [36] and the formalism introduced will also be further developed to include phase to phase turbulent interactions as proposed in [34]. All of this will allow more predictive simulations and validations against measurements for spray and vapor distribution in high-pressure test cells.

- The kinetic approach, as it was presented here, is well designed to compute dilute and moderately dense spray regions. Another important evolution will consist in extending the model to higher spray density regions where other physical mechanisms must be considered. This challenge will be addressed in the framework of reduced order models in order to account for all the computational aspects of the injection application, as it was introduced in the general context of two-phase flows modeling. Let us also recall that some preliminary works to describe the whole injection processus through Eulerian methods have been recently initiated in [68], in the spirit of coupling kinetic based Eulerian polydisperse methods with a diffuse interface method for the liquid atomization.

\section{Acknowledgements}

The main contribution of this review paper relies on the Ph.D. works conducted by D. Kah (2007-2010) and O. Emre (2010-2014). These Ph.D. are the result of a fruitful collaboration between IFPEN and EM2C Laboratory at Ecole Centrale Paris and were co-advised by S. Jay and S. de Chaisemartin at IFPEN and F. Laurent-Nègre and M. Massot at Ecole Centrale Paris in collaboration with Rodney O. Fox. The research of R.O.F. leading to some of the results reported in the present work on quadrature-based moment methods and turbulence modeling has received funding from the European Union Seventh Framework Program (FP7/2007-2013) under grant agreement No. 246556. 


\section{References}

[1] B. Abramzon and W. A. Sirignano. Droplet vaporisation model for spray combustion calculations. International Journal of Heat and Mass Transfer, 9:1605-1618, 1989.

[2] A.A. Amsden, P. J. O'Rourke, and T. D. Butler. KIVA II, a computer program for chemically reactive flows with sprays. Technical Report LA-11560-MS, Report Los Alamos National Laboratory, Los Alamos, New Mexico, 1989.

[3] M. R. Baer and J. W. Nunziato. A two-phase mixture theory for the deflagration-to-detonation transition (DDT) in reactive granular materials. International journal of multiphase flow, 12(6):861-889, 1986.

[4] S. Balachandar and John K. Eaton. Turbulent dispersed multiphase flow. Ann. Rev. Fluid Mech., 42(1):111133, 2010.

[5] H. Belaid-Saleh. Etude expérimentale et numérique des régimes de combustion dans le contexte Dual-Fuel. $\mathrm{PhD}$, Université d'Orléans, 2015.

[6] H. Belaid-Saleh, S. Jay, J. Kashdan, C. Ternel, and C. Mounaim-Rousselle. Numerical and experimental investigation of combustion regimes in a dual fuel engine. SAE Technical Paper, (2013-24-0015), 092013.

[7] R. BenDakhlia. Combustion stationnaire et instationnaire de mélanges diphasiques. PhD thesis, Ecole Centrale Paris, 2001.

[8] G. A. Bird. Molecular gas dynamics and the direct simulation of gas flows. Oxford Science Publications, 42, 1994.

[9] J. Bohbot, N. Gillet, and A. Benkenida. IFP-C3D: An unstructured parallel solver for reactive compressible gas flow with spray. Oil and Gas Science and Technology, 64(3):309-355, 2009.

[10] M. Boileau. Simulation aux grandes échelles de l'allumage diphasique des foyers aéronautiques. PhD thesis, Institut National Polytechnique de Toulouse, 2007.

[11] F. Bouchut, S. Jin, and X. Li. Numerical approximations of pressureless and isothermal gas dynamics. SIAM J. Num. Anal., 41:135-158, 2003.

[12] S. Candel and T. Poinsot. Flame stretch and the balance equation for the flame area. Combustion Science and Technology, 70:1-15, 1990.

[13] J. Capecelatro and O. Desjardins. An Euler-Lagrange strategy for simulating particle-laden flows. J. Comp. Phys., 238:1-31, 2013.

[14] C. Chalons, R. O. Fox, F. Laurent, M. Massot, and A. Vié. A multi-Gaussian quadrature-based moment method for dispersed multiphase flows. Submitted to SIAM Multiscale Modeling and Simulation, available on $H A L, 2014$.

[15] C. Chalons, R. O. Fox, and M. Massot. A multi-Gaussian quadrature method of moments for gas-particle flows in a LES framework. In Proceedings of the Summer Program 2010, Center for Turbulence Research, Stanford University, pages 347-358, Stanford, 2010.

[16] C. Chalons, D. Kah, and M. Massot. Beyond pressureless gas dynamics: quadrature-based velocity moment models. Communication in Mathematical Sciences, 10(4):1241-1272, 2012.

[17] S. Chapman and T. G. Cowling. The Mathematical Theory of Non-Uniform Gases. Cambridge Mathematical Library, 1970.

[18] V. Le Chenadec and H. Pitsch. A 3D unsplit forward/backward volume-of-fluid approach and coupling to the level set method. Journal of Computational Physics, 233(0):10 - 33, 2013.

[19] V. Le Chenadec and H. Pitsch. A monotonicity preserving conservative sharp interface flow solver for high density ratio two-phase flows. Journal of Computational Physics, 249(0):185 - 203, 2013.

[20] S. de Chaisemartin. Eulerian models and numerical simulation of turbulent dispersion for polydisperse evaporation sprays. PhD thesis, Ecole Centrale Paris, France, 2009. http://tel.archives-ouvertes.fr/tel00443982/en/.

[21] F.-X. Demoulin, P.A. Beau, G. Blokkeel, A. Mura, and R. Borghi. A new model for turbulent flows with large density fluctuations: application to liquid atomization. Atomization and Sprays, 17(4):315-345, 2007.

[22] F.-X. Demoulin, G. Blokkeel, A. Mura, P.A. Beau, and R. Borghi. Modeling of liquid atomization as a turbulent flow with high density fluctuations. Atomization and Sprays, 17:298-313, 2007.

[23] H. Dette and W. J. Studden. The theory of canonical moments with applications in statistics, probability, and analysis. Wiley Series in Probability and Statistics: Applied Probability and Statistics. John Wiley \& Sons Inc., New York, 1997. A Wiley-Interscience Publication.

[24] F. Doisneau. Eulerian modeling and simulation of polydisperse moderately dense coalescing spray flows with nanometric-to-inertial droplets: Application to Solid Rocket Motors. PhD, Ecole Centrale Paris, 2013. https://tel.archives-ouvertes.fr/tel-01009896. 
[25] F. Doisneau, F. Laurent, A. Murrone, J. Dupays, and M. Massot. Optimal Eulerian model for the simulation of dynamics and coalescence of alumina particles in solid propellant combustion. In Proceedings of the 7th International Conference on Multiphase Flows, ICMF 2010, pages 1-15, Tampa - Florida USA, 2010.

[26] F. Doisneau, F. Laurent, A. Murrone, J. Dupays, and M. Massot. Evaluation of Eulerian Multi-Fluid models for the simulation of dynamics and coalescence of particles in solid propellant combustion. Journal of Computational Physics, 234:230-262, 2013.

[27] F. Doisneau, A. Sibra, J. Dupays, A. Murrone, F. Laurent, and M. Massot. An efficient and accurate numerical strategy for two-way coupling in unsteady polydisperse moderately dense sprays: application to Solid Rocket Motor instabilities. Journal of Propulsion and Power, 30(3):727-748, 2014.

[28] F. Doisneau, O. Thomine, F. Laurent, A. Vié, and M. Massot. Eulerian modeling and simulation of small scale trajectory crossing and coalescence for moderate-stokes-number spray flows. In Volume du «Summer Program 2012», publication du Center for Turbulence Research, pages 365-374, Stanford University, 2012.

[29] D. A. Drew. Mathematical modeling of two-phase flow. Ann. Rev. Fluid. Mech., 15:261-91, 1983.

[30] G. Dufour. Modélisation multi-fluide eulérienne pour les écoulements diphasiques à inclusions dispersées. PhD thesis, Université Paul Sabatier Toulouse III, 2005.

[31] J. K. Dukowicz. A particle-fluid numerical model for liquid sprays. J. Comput. Phys., 35(2):229-253, 1980.

[32] C. Dumouchel. On the experimental investigation of primary atomization of liquid streams. Experiments in Fluids, 45(3):371-422, 2008.

[33] O. Emre. Modeling of spray polydispersion with two-way turbulent interactions for high pressure direct injection in engines. PhD, Ecole Centrale Paris, 2014.

[34] O. Emre, R.O Fox, M. Massot, S. de Chaisemartin, S. Jay, and F. Laurent. Eulerian modeling of a polydisperse evaporating spray under realistic internal-combustion-engine conditions. Flow, Turbulence and Combustion, 93(4):689-722, 2014. http://hal.archives-ouvertes.fr/hal-00942115.

[35] O. Emre, F. Laurent, S. de Chaisemartin, S. Jay, S. Kah, and M. Massot. Two-way coupling modeling through eulerian moment method for spray injection in engine simulations. In European Congress on Computational Methods in Applied Sciences and Engineering (ECCOMAS2012), Vienna, Austria, 2012.

[36] O. Emre, F. Laurent, S. de Chaisemartin, S. Jay, and M. Massot. Numerical analysis of a two-way coupled Eulerian high order moment mthod for the simulation of polydisperse evaporating sprays. in preparation, 2014.

[37] V. Ferrand, R. Basile, and J. Borée. Gas-droplet turbulent velocity correlations and two-phase interaction in an axisymmetric jet laden with partly responsive droplets. International journal of multiphase flow, 29:195217, 2003.

[38] P. Fevrier, O. Simonin, and K. Squires. Partitioning of particle velocities in gas-solid turbulent flows into a continuous field and a spatially uncorrelated random distribution theoretical formalism and numerical study. Journal of Fluid Mechanics, 533:1-46, 2005.

[39] R. O. Fox. Introduction and fundamentals of modeling approaches for polydisperse multiphase flows. In Multiphase reacting flows: modelling and simulation, volume 492 of CISM Courses and Lectures, pages 1-40. Springer Wien, 2007. Editors D.L. Marchisio and R. O. Fox, Udine, July 2006.

[40] R. O. Fox. A quadrature-based third-order moment method for dilute gas-particle flow. Journal of Computational Physics, 227(12):6313-6350, 2008.

[41] R. O. Fox, F. Laurent, and M. Massot. Numerical simulation of spray coalescence in an Eulerian framework: direct quadrature method of moments and multi-fluid method. J. Comput. Phys., 227(6):3058-3088, 2008.

[42] Rodney O. Fox. On multiphase turbulence models for collisional fluid-particle flows. Journal of Fluid Mechanics, 742:368-424, 32014.

[43] E. Franquet and V. Perrier. Runge-kutta discontinuous galerkin method for interface flows with a maximum preserving limiter. Computers \& Fluids, 65:2-7, 2012.

[44] E. Franquet and V. Perrier. Runge-kutta discontinuous galerkin method for the approximation of baer and nunziato type multiphase models. J. Comput. Phys., 231:4096-4141, 2012.

[45] L. Freret, S. de Chaisemartin, F. Laurent, P. Vedula, R. O. Fox, O. Thomine, J. Reveillon, and M. Massot. Eulerian moment models for polydisperse weakly collisional sprays : model and validation. In Proceedings of the Summer Program 2008, Center for Turbulence Research, Stanford University, pages 277-288, 2009.

[46] M. Garcia. Développement et validation du formalisme Euler-Lagrange dans un solveur parallèle nonstructuré pour la simulation aux grandes échelles. PhD thesis, Institut National Polytechnique de Toulouse, 2009.

[47] J. B. Greenberg, I. Silverman, and Y. Tambour. On the origin of spray sectional conservation equations. Combustion and Flame, 93:90-96, 1993. 
[48] C. Habchi. Modélisation Tridimensionnelle de l'injection et de la préparation du mélange gaz/carburant dans les moteurs à combustion interne. PhD thesis, Institut National Polytechnique de Toulouse, 2006.

[49] E. Hairer and G.Wanner. Solving Ordinary Differential Equations II, volume 2. Springer Series In Computational Mathematics, 1996.

[50] M. Herrmann. A Eulerian level set/vortex sheet method for two-phase interface dynamics. J. Comput. Phys., 203(2):539-571, 2005

[51] M. Herrmann. A balanced force refined level set grid method for two-phase flows on unstructured flow solver grids. Journal of Computational Physics, 227(4):2674 - 2706, 2008.

[52] John B. Heywood. Internal Combustion Engine Fundamentals. McGraw-Hill series in mechanical engineering, 1988.

[53] C. W. Hirt, A. A. Amsden, and J. L. Cook. An arbitrary Lagrangian-Eulerian computing method for all flow speeds. Journal of Computational Physics, 14(3):227-253, 1974.

[54] M. Ishii. Thermo-Fluid dynamic theory of two-phase flow. Eyrolles, 1975.

[55] S. Jay, F. Lacas, and S. Candel. Combined surface density concepts for dense spray combustion. Combustion and Flame, 144(3):558-577, 2006.

[56] M. Juniper, A. Tripathi, P. Scouflaire, C. Rolon, and S. Candel. Proceedings of the Combustion Institute, pages 1103-1109, 2001.

[57] D. Kah. Prise en compte des aspects polydispersés pour la modélisation d'un jet de carburant dans les moteurs à combustion interne. PhD, Ecole Centrale Paris, http://tel.archives-ouvertes.fr/ tel-00618786, 2010.

[58] D. Kah, O. Emre, Q.H. Tran, S. de Chaisemartin, S. Jay, F. Laurent, and M. Massot. High order moment method for polydisperse evaporating spray with mesh movement : application to internal combustion engines. To appear in International Journal of Multiphase Flows, 2015. http://hal.archives-ouvertes.fr/hal00942115.

[59] D. Kah, F. Laurent, M. Massot, and S. Jay. Modeling of polydisperse sprays using a high order size moment method for the numerical simulation of advection and evaporation. In Proceedings of the 11th ICLASS, International Conference on Liquid Atomization and Spray Systems Vail, Colorado, 2009.

[60] D. Kah, F. Laurent, M. Massot, and S. Jay. A high order moment method simulating evaporation and advection of a polydisperse liquid spray. J. Comput. Phys., 231(2):394-422, 2012.

[61] N. Lamarque. Schémas numériques et conditions limites pour la simulation aux grandes échelles de la combustion diphasique dans les foyers d'hélicoptère. PhD thesis, Institut National Polytechnique de Toulouse, 2007.

[62] F. Laurent. Numerical analysis of Eulerian multi-fluid models in the context of kinetic formulations for dilute evaporating sprays. M2AN Math. Model. Numer. Anal., 40(3):431-468, 2006.

[63] F. Laurent and M. Massot. Multi-fluid modeling of laminar poly-dispersed spray flames: origin, assumptions and comparison of the sectional and sampling methods. Comb. Theory and Modelling, 5:537-572, 2001.

[64] F. Laurent, M. Massot, and P. Villedieu. Eulerian multi-fluid modeling for the numerical simulation of coalescence in polydisperse dense liquid sprays. J. Comput. Phys., 194:505-543, 2004.

[65] F. Laurent, A. Sibra, and F. Doisneau. Two-size moment eulerian multi-fluid model for polydisperse sprays: a flexible and efficient computational approach, in preparation. 2014.

[66] F. Laurent, A. Vié, C. Chalons, R.O. Fox, and M. Massot. A hierarchy of Eulerian models for trajectory crossing in particle-laden turbulent flows over a wide range of Stokes numbers. In Annual Research Brief of the Center for Turbulence Research, Center for Turbulence Research, Stanford University, pages 193-204, 2012.

[67] S. Le Martelot. Contribution à la simulation numérique directe de l'ébullition. PhD thesis, Aix Marseille Université, 2013.

[68] C. Le Touze, A. Murrone, and E. Montreuil. Numerical coupling strategies for the 'separated-to-dispersed' transition within the liquid phase of cryogenic rocket engines. In $5^{\text {th }}$ european conference for aeronautics and space sciences, 2013.

[69] R. Lebas, T. Menard, P. A. Beau, A. Berlemont, and F. X. Demoulin. Numerical simulation of primary break-up and atomization: DNS and modelling study. Int. J. Multiphase Flows, 35(3):247-260, MAR 2009.

[70] R. J. LeVeque. Numerical methods for conservation laws. Birkhäuser Verlag, Basel, second edition, 1992.

[71] D. Lhuillier. A mean-field description of two-phase flows with phase changes. International Journal of Multiphase Flows, 23:511-525, 2003.

[72] R. L. Libo. Kinetic Theory: Classical, Quantum, and Relativistic Descriptions, volume 2 of Prentice-Hall Advanced Reference Series. London, 1998. 
[73] E. Loth. Numerical approaches for motion of dispersed particles, droplets and bubbles. Prog. Energy and Comb. Sci., 26:161-223, 2000.

[74] B. Mandumpala Devassy. Atomization modelling of liquid jets using an Eulerian-Eulerian model and a surface density approach. PhD thesis, Aix Marseille Université, 2013.

[75] F.E. Marble and J.E. Broadwell. The coherent flame model for turbulent chemical reactions. Technical Report Tech. Rep. TRW-9-PU, Project Squid, 1977.

[76] D. L. Marchisio and R. O. Fox. Solution of population balance equations using the direct quadrature method of moments. Journal of Aerosol Science, 36:43-73, 2005.

[77] L. Martinez, J.-B. Michel, S. Jay, and O. Colin. Evaluation of different tabulation techniques dedicated to the prediction of the combustion and pollutants emissions on a diesel engine with 3D CFD. SAE Technical Paper, (2013-01-1093), 042013.

[78] L. Martinez, A. Vié, S. Jay, A. Benkenida, and B. Cuenot. Large Eddy Simulation of Fuel sprays using the Eulerian Mesoscopic Approach. Validations in realistic engine conditions. In ICLASS, 2009.

[79] E. Masi and O. Simonin. An algebraic-closure-based moment method for unsteady eulerian modeling of non-isothermal particle-laden turbulent flows in very dilute regime and high stokes number. In 7 th Int. Symposium on Turbulence, Heat and Mass Transfer, Palermo, IT, 2012.

[80] E. Masi, O. Simonin, E. Riber, P. Sierra, and L.Y.M. Gicquel. Development of an algebraic-closure-based moment method for unsteady Eulerian simulations of particle-laden turbulent flows in very dilute regime. International Journal of Multiphase Flow, 58(0):257 - 278, 2014.

[81] M. Massot, R. Knikker, C. Péra, and J. Réveillon. Lagrangian/Eulerian analysis of the dispersion of evaporating droplets in a non-homogeneous turbulent flow. In Proceedings of ICMF'04, 2004.

[82] M. Massot, F. Laurent, S. de Chaisemartin, L. Fréret, and D. Kah. Eulerian multi-fluid models: modeling and numerical methods. In Modelling and Computation of Nanoparticles in Fluid Flows, Lectures of the von Karman Institue, pages 1-86. NATO RTO AVT 169, 2009. https://www.cso.nato.int/Pubs/rdp.asp?RDP=RTO-EN-AVT-169, available on HAL : http://hal.archivesouvertes.fr/hal-00423031/en/.

[83] M. Massot, F. Laurent, D. Kah, and S. de Chaisemartin. A robust moment method for evaluation of the disappearance rate of evaporating sprays. SIAM J. Appl. Math., 70:3203-3234, 2010. http://hal.archivesouvertes.fr/hal-00332423/en/.

[84] R. McGraw. Description of aerosol dynamics by the quadrature method of moments. Aerosol Science and Technology, 27:255-265, 1997.

[85] R. McGraw. Numerical advection of correlated tracers: preserving particle size/composition moment sequences during transport of aerosol mixtures. J. Phys.: Conf. Ser., 78(012045):1-5, 2007.

[86] L. R. Mead and N. Papanicolaou. Maximum entropy in the problem of moments. J. Math. Phys., 25(8):24042417, 1984.

[87] T. Menard, S. Tanguy, and A. Berlemont. Coupling level set/VOF/ghost fluid methods: Validation and application to 3D simulation of the primary break-up of a liquid jet. International Journal of Multiphase Flow, 33(5):510-524, 2007.

[88] O. Le Métayer. Modélisation et résolution de la propagation de fronts perméables. Application aux fronts d'évaporation et de détonation. PhD thesis, Université de Provence, 2003.

[89] R. S. Miller and J. Bellan. Direct numerical simulation of a confined three-dimensional gas mixing layer with one evaporating hydrocarbon-droplet-laden stream. Journal of Fluids Mechanics, 384:293-338, 1999.

[90] C. Morel. Modélisation multidimensionnelle des écoulements diphasiques gaz-liquide. Application à la simulation des écoulements à bulles ascendants en conduite verticale. PhD thesis, Ecole Centrale Paris, 1997.

[91] J.-B. Mossa. Extension polydisperse pour la description euler-euler des écoulements diphasiques réactifs TH/CFD/05/74. PhD thesis, Institut National Polytechnique de Toulouse, 2005.

[92] A. Murrone and P. Villedieu. Numerical modeling of dispersed two-phase flows. Technical report, Aerospace Lab 2, 2011.

[93] P. J. O'Rourke. Collective drop effects on vaporizing liquid sprays. PhD thesis, Princeton University, 1981.

[94] M. Pai and S. Subramaniam. Two-way coupled stochastic model for dispersion of inertial particles in turbulence. Journal of Fluid Mechanics, 700:29-62, 2012.

[95] L.M. Pickett, J. Manin, C.L. Genzale, D.L. Siebers, M.P.B. Musculus, and C.A. Idicheria. Relationship between diesel fuel spray vapor penetration/dispersion and local fuel mixture fraction. SAE Int. J. Engines, 2011.

[96] N. Pottier. Physique statistique hors d'équilibre - Processus irréversibles linéaires. EDP Sciences, 2007.

[97] J. Reveillon. Direct numerical simulation of sprays: turbulent dispersion evaporation and combustion. In 
Multiphase reacting flows: modelling and simulation, volume 492 of CISM Courses and Lectures, pages 229-273. Springer Wien, 2007. Editors D.L. Marchisio and R. O. Fox, Udine, July 2006.

[98] J. Reveillon, N.C. Bray, and L. Vervish. DNS study of spray vaporization and turbulent micro-mixing. In AIAA 98-1028, 36th Aerospace Sciences Meeting and Exhibit, Reno NV, 1998.

[99] J. Reveillon and F.-X. Demoulin. Effects of the preferential segregation of droplets on evaporation and turbulent mixing. J. Fluid Mech, 583:273-302, 2007.

[100] E. Riber, M. Garcia, V. Moureau, H. Pitsch, O. Simonin, and T. Poinsot. Evaluation of numerical strategies for LES of two-phase reacting flows. Proceedings of the Summer Program 2006, Center for Turbulence Research, Stanford University, 2006.

[101] J. J Riley and G. S. Patterson. Diffusion experiments with numerically intergrated isotropic turbulence. Physics of Fluids, 17:292-297, 1974.

[102] P. Rosin and E. Rammler. Laws governing the fineness of powdered coal. J. Inst. Fuel, (7):29-36, 1933.

[103] M. Rüger, S. Hohmann, M. Sommerfeld, and G. Kohnen. Euler/Lagrange calculations of turbulent sprays: the effect of droplet collisions and coalescence. Atomization and Sprays, 10(1):47-81, 2000.

[104] R. Saurel and R. Abgrall. A multiphase Godunov method for compressible multifluid and multiphase flow. Journal of Computational Physics, 150:425-467, 1999.

[105] P. Sierra. Modeling the dispersion and evaporation of sprays in aeronautical combustion chambers. $\mathrm{PhD}$ thesis, INP Toulouse, 2012.

[106] I. Silverman, J. B. Greenberg, and Y. Tambour. Asymptotic analysis of a premixed polydisperse spray flame. SIAM J. Appl. Math., 51(5):1284 - 1303, 1991.

[107] R.M.C. So, L.H. Jin, and T.B. Gatski. An explicit algebraic Reynolds stress and heat flux model for incompressible turbulence: Part I Non-isothermal flow. Theoretical and Computational Fluid Dynamics, 17(56):351-376, 2004.

[108] H. Struchtrup. Macroscopic transport equations for rarefied gas flows. Interaction of Mechanics and Mathematics. Springer, Berlin, 2005. Approximation methods in kinetic theory.

[109] S. Subramaniam. Statistical representation of a spray as a point process. Physics of Fluids, 12:2413, 2000.

[110] S. Subramaniam. Statistical modeling of sprays using the droplet distribution function. Physics of Fluids, 13:624, 2001.

[111] A. Tagliani. Hausdorff moment problem and maximum entropy: a unified approach. Appl. Math. Comput., 105(2-3):291-305, 1999.

[112] Y. Tambour. A sectional model for evaporation and combustion of sprays of liquid fuel. Isr. J. Technol., $18: 47-56,1980$.

[113] E. F. Toro. Riemann solvers and numerical methods for fluid dynamics. Springer, 2009.

[114] B. Truchot. Développement et validation d'un modèle eulérien en vue de la simulation des jets de carburants dans les moteurs à combustion interne. PhD thesis, Institut National Polytechnique de Toulouse, 2005.

[115] S. Unverdi and G. Tryggvason. A front-tracking method for viscous incompressible multi-fluid flows. $J$. Comput. Phys., 100:25-37, 1992.

[116] A. Vallet, A. Burluka, and R. Borghi. Development of a eulerian model for the "atomization" of a liquid jet. Atomization and Sprays, 11:619-642, 2001.

[117] C. Vessiller. Contribution à l'étude des brouillards denses et dilués par la simulation numérique Euler/Euler et Euler/Lagrange. PhD thesis, Ecole Centrale Paris, 2007.

[118] A. Vié, F. Doisneau, and M. Massot. On the Anisotropic Gaussian closure for the prediction of inertialparticle laden flows. Communications in Computational Physics, 17(1):1-46, 2015. http://hal.archivesouvertes.fr/hal-00912319.

[119] A. Vié, E. Masi, O. Simonin, and M. Massot. On the direct numerical simulation of moderate-Stokesnumber turbulent particulate flows using Algebraic-Closure-Based and Kinetic-Based Moment Methods. In Proceedings of the Summer Program 2012, Center for Turbulence Research, Stanford University, pages 355-364, 2012.

[120] C. Villani. A review of mathematical topics in collisional kinetic theory. S. Friedlander and D. Serre Eds., 2002. Handbook of Fluid Mechanics.

[121] A. Vié. Simulation aux grandes échelles d'écoulements diphasiques turbulents à phase liquide dispersée. PhD thesis, Université Paul Sabatier Toulouse III, 2010.

[122] A. Vié, F. Laurent, and M. Massot. Size velocity correlations in high order moment methods for polydisperse evaporating sprays : modeling and numerical issues. Journal of Computational Physics, 237:177-210, 2013.

[123] F. A. Williams. Spray combustion and atomization. Phys. Fluids, 1:541-545, 1958.

[124] F. A. Williams. Combustion Theory (Combustion Science and Engineering Series). ed F A Williams (Read- 
ing, MA: Addison-Wesley), 1985.

[125] D. L. Wright. Numerical advection of moments of the particle size distribution in Eulerian models. Journal of Aerosol Science, 38(3):352-369, 2007.

[126] D. L. Wright, R. McGraw, and D. E. Rosner. Bivariate extension of the quadrature method of moments for modeling simultaneous coagulation and sintering of particle populations. J. of Colloid and Interface Sci., 236:242-251, 2001.

[127] C. Yuan and R. O. Fox. Conditional quadrature method of moments for kinetic equations. Journal of Computational Physics, 230:8216-8246, 2011.

[128] C. Yuan, F. Laurent, and R. O. Fox. An extended quadrature method of moments for population balance equations. J. Aerosol Sci., 51:1-23, 2012.

[129] L. I. Zaichik, O. Simonin, and V. M. Alipchenkov. An Eulerian approach for large eddy simulation of particle transport in turbulent flows. Journal of Turbulence, 10(4):1468-5248, 2009.

[130] H.P. Zhu, Z.Y. Zhou, R.Y. Yang, and A.B. Yu. Discrete particle simulation of particulate systems: Theoretical developments. Chemical Engineering Science, 62(13):3378 - 3396, 2007. 